\title{
THE SOUTHERN ARGENTINA AGILE METEOR RADAR ORBITAL SYSTEM (SAAMER-OS): AN INITIAL SPORADIC METEOROID ORBITAL SURVEY IN THE SOUTHERN SKY
}

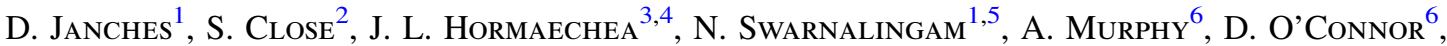 \\ B. VAndepeer ${ }^{6}$, B. Fuller ${ }^{6}$, D. C. Fritts $^{7}$, And C. Brunini ${ }^{8}$ \\ ${ }^{1}$ Space Weather Laboratory, Mail Code 674, NASA Goddard Space Flight Center, Greenbelt, MD 20771, USA; \\ diego.janches@nasa.gov, nimalan.swarnalingam@nasa.gov \\ ${ }^{2}$ Space Environment and Satellite Systems Laboratory, Department of Aeronautics and Astronautics, Stanford University, Palo Alto, CA, USA; sigridc@ stanford.edu \\ ${ }^{3}$ Estacion Astronomica Rio Grande, Rio Grande, Tierra del Fuego, Argentina; jlhormaechea@ untdf.edu.ar \\ ${ }^{4}$ Universidad Nacional de Tierra del Fuego, Tierra del Fuego, Argentina; \\ ${ }^{5}$ Department of Physics, Catholic University of America, Washington, DC 20064, USA; \\ ${ }^{6}$ Genesis Software Pty Ltd, Adelaide, Australia; amurphy@gsoft.com.au, doconnor@gsoft.com.au, bvandepe@gsoft.com.au, bfuller@gsoft.com.au \\ GATS Inc., Boulder CO, USA; dave@gats-inc.com \\ ${ }^{8}$ Departmento de Astronomia y Geofísica, Universidad Nacional de La Plata, La Plata, Argentina; claudiobrunini@yahoo.com \\ Received 2015 April 25; accepted 2015 July 5; published 2015 August 6
}

\begin{abstract}
We present an initial survey in the southern sky of the sporadic meteoroid orbital environment obtained with the Southern Argentina Agile MEteor Radar (SAAMER) Orbital System (OS), in which over three-quarters of a million orbits of dust particles were determined from 2012 January through 2015 April. SAAMER-OS is located at the southernmost tip of Argentina and is currently the only operational radar with orbit determination capability providing continuous observations of the southern hemisphere. Distributions of the observed meteoroid speed, radiant, and heliocentric orbital parameters are presented, as well as those corrected by the observational biases associated with the SAAMER-OS operating parameters. The results are compared with those reported by three previous surveys performed with the Harvard Radio Meteor Project, the Advanced Meteor Orbit Radar, and the Canadian Meteor Orbit Radar, and they are in agreement with these previous studies. Weighted distributions for meteoroids above the thresholds for meteor trail electron line density, meteoroid mass, and meteoroid kinetic energy are also considered. Finally, the minimum line density and kinetic energy weighting factors are found to be very suitable for meteroid applications. The outcomes of this work show that, given SAAMER's location, the system is ideal for providing crucial data to continuously study the South Toroidal and South Apex sporadic meteoroid apparent sources.
\end{abstract}

Key words: meteorites, meteors, meteoroids - techniques: radar astronomy - zodiacal dust

\section{INTRODUCTION}

The solar system, like other systems (e.g., the "Vega Phenomenon"; Beichman 1987), contains a thick circumsolar disk of dust and small debris known as the Zodiacal Dust Cloud (ZDC). The dust originates from a variety of sources, such as asteroid collisions, comet disintegration and activity (Jenniskens 2006; Nesvorný et al. 2010, 2011b), and a minority from the interstellar medium. This cloud, which is believed to inject between $\sim 5$ and $\sim 300 \mathrm{td}^{-1}$ (metric tons per day; Plane 2003, 2012; Plane et al. 2015) of material into the Earth's upper atmosphere, plays a significant role not only in its composition but also in the Earth's orbital environment. The continuous bombardment of the Earth by these small, highly energetic interplanetary dust particles presents a collision threat to spacecraft (Close et al. 2000, 2010), as well as providing the dominant source of metallic atoms in the mesosphere and lower thermosphere (MLT; Plane 2012; Feng et al. 2013; Marsh et al. 2013; Janches et al. 2014b). Most of the ZDC particles form the so-called sporadic meteoroid complex (SMC), and unlike meteor showers, their orbits have evolved significantly over time. This evolution is the product of effects such as PoyntingRobertson (PR) drag, radiation pressure, sublimation, mutual collisions, and the dynamical effects of the planets (Backman 1997) and results in sporadic meteoroids exhibiting a wide distribution of physical properties, in particular those of mass, speed, and orbital parameters. A consequence of this is that it is unlikely to associate sporadic meteors with any particular parent body, and thus they can only be characterized through statistical measurements of the SMC's interaction with the Earth.

The majority of the incoming meteoroid flux originating from the ZDC is in the mass range of $10^{-11}-10^{-4} \mathrm{~g}$ and enters the atmosphere at speeds between 11 and $72 \mathrm{~km} \mathrm{~s}^{-1}$ (Hughes 1978; Wasson \& Kyte 1987; Ceplecha et al. 1998; Love \& Brownlee 1993; Cziczo et al. 2001; Mathews et al. 2001; Gabrielli et al. 2004; Plane 2004; Lanci \& Kent 2006; Lanci et al. 2007; Fentzke \& Janches 2008; Nesvorný et al. 2010, 2011a; Gardner et al. 2011). The highly energetic collisions with air molecules cause the meteoroids to heat and ablate, releasing both neutral and ionized atoms from the meteoroid. These phenomena associated with a meteoroid impacting a planetary atmosphere are collectively termed a meteor (i.e., a plasma) ${ }^{9}$ and are typically observable by an assorted class of ground-based radars (Janches et al. 2003, 2014a). All-sky VHF meteor radars, such as the Southern Argentina Agile MEteor Radar (SAAMER), based their studies mainly on underdense specular meteor trails, which are generally semi-stationary plasma columns left in the wake of the meteoroid's trajectory (Baggaley 2002). The advantage of using such systems is that

\footnotetext{
9 Limiting Future Collision Risk to Spacecraft: An Assessment of NASA's Meteoroid and Orbital Debris Programs (2011). Authors: Committee for the Assessment of NASA's Orbital Debris Programs; Aeronautics and Space Engineering Board; Division on Engineering and Physical Sciences; National Research Council.
} 
they are generally dedicated to meteor observations and over time are capable of collecting large data sets of meteoroid statistics covering various conditions, namely, the seasonal and diurnal changes to the Earth's orientation and location in space.

Over the past 50 years, three meteor radar systems were used for detailed orbital surveys of the SMC: (1) the Harvard Radio Meteor Project (HRMP; Hawkins 1963; Jones \& Brown 1993; Taylor \& Elford 1998; Hunt et al. 2004), a scientific investigative station based within Harvard University (USA); (2) the Advanced Meteor Orbit Radar (AMOR; Baggaley et al. 1994; Galligan \& Baggaley 2004, 2005), a system that operated in Christchurch, New Zealand; and (3) the Canadian Meteor Orbit Radar (CMOR; Webster et al. 2004; Jones et al. 2005; Campbell-Brown 2008; Campbell-Brown \& Wiegert 2009; Brown et al. 2010; Wiegert et al. 2011), which is currently operating in Ontario, Canada. The HRMP observations provided about $2 \times 10^{4}$ meteoroid orbits from 1968 to 1969 and have been used as the basis for many of the existing near-Earth meteoroid stream searches (Sekanina 1973, 1976) and environment models (Drolshagen et al. 2008). A much larger study was conducted by AMOR, which observed approximately $5 \times 10^{5}$ orbits over the course of 5 years from 1995 to 1999 (Galligan \& Baggaley 2004, 2005). Additionally, AMOR was located in the Southern Hemisphere, and although the radar is no longer operational, it has provided the most detailed study of meteoroids in this region of the sky to date. The still-operational CMOR system has recorded 3 million individual meteoroid orbits for particles with mean mass near $10^{-7} \mathrm{~kg}$ (radio magnitude +7.5 ; Verniani 1973) from 2002 to 2008 (Brown et al. 2008, 2010; Campbell-Brown 2008), and each day $\sim 4000-5000$ are added to the statistics.

The SAAMER Orbital System (SAAMER-OS) is the latest meteor radar conducting continuous meteor observations. Initially, SAAMER was a single station radar system. In 2010 August, the system was upgraded by adding two remote receiving stations and thus making it capable of determining meteor orbits. SAAMER-OS has been fully operational and recording orbits since 2012 January. Despite experiencing 6 months of interruption in 2013 due to technical problems that have now been mostly addressed and some low detection rates in 2014 due to interference, a data set that comprises more than 750,000 orbits is available as of 2015 April for an initial study, including a comparison with the previous orbital meteor radar investigations. The aim of this manuscript is to present SAAMER's initial results and discuss them on the framework of past surveys. To achieve this, a general description of the SAAMER system, as well as the methodology used for meteoroid orbit determination and observational bias correction, is described in Section 2. The different corrections applied to the observed results are described in Section 3. The observed and corrected results are presented in Section 4, along with a comparison with previous reported surveys. In Section 5 specific results for the south toroidal source are presented. The conclusions are presented in Section 6.

\section{SAAMER ORBITAL SYSTEM AND METHODOLOGY}

\subsection{System Description}

The SAAMER-OS is hosted by the Estacion Astronómica Rio Grande (EARG), located in Rio Grande, Tierra del Fuego, Argentina. It consists of three distinct radar stations: the central station (SAAMER-C; 53 :786 S, 67.751 W), which hosts the

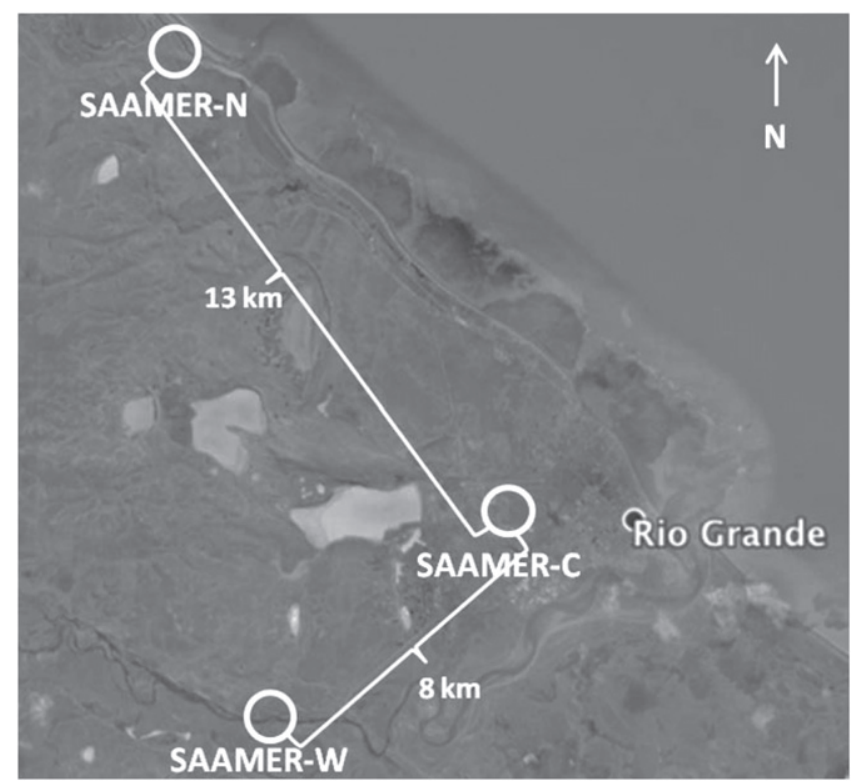

Figure 1. SAAMER-OS geographic layout. Rio Grande is a city in Tierra del Fuego, Argentina, located at a geographical latitude of $\sim 53.8 \mathrm{~S}$ and longitude of $67^{\circ} .7 \mathrm{~W}$.

transmitting and interferometry-enabled receiving antenna arrays; the northern remote station (SAAMER-N; 53:682 S, $67.871 \mathrm{~W}$ ), located approximately $13 \mathrm{~km}$ northwest of the central station; and the western remote station (SAAMER-W; $53.828 \mathrm{~S}, 67.842 \mathrm{~W}$ ), located approximately $8 \mathrm{~km}$ southwest of the central station. The geographic layout of SAAMER-OS is shown in Figure 1.

SAAMER-C has been in operation since 2008 May and was designed to enable gravity wave momentum flux measurements in the MLT (Fritts et al. 2010a, 2010b). This requirement led to the use of a rather high peak transmitter power $(60 \mathrm{~kW})$ relative to specular meteor radars (6-20 kW; W. Hocking 2015, private communication; Fritts et al. 2012), as well as an eight-antenna transmitter array, designed by Mardoc Inc., composed of threeelement crossed yagi antennas as opposed to the typical design of a single, two-element crossed yagi transmit antenna. The transmitting array is organized in a circular pattern of diameter $27.6 \mathrm{~m}$ (i.e., three times the radar wavelength), and the phase differences among transmitting antennas can be changed electronically, adding flexibility to the system to perform a number of transmitting and receiving modes (Figure 3; Janches et al. 2014a). In normal operation mode each transmitting antenna transmits at a phase difference of $180^{\circ}$ from the adjoining two antennas (i.e., every other antenna has the same phase). This provides a gain pattern in which the majority of the power is focused into eight beams at $45^{\circ}$ azimuth increments with peak power at approximately $35^{\circ}$ off zenith (Figure 2). The resulting transmit gain pattern causes the majority of the meteor detections to occur between $15^{\circ}$ and $50^{\circ}$ off zenith. The system parameters utilized for the continuous mode of operation, driven by the mesospheric dynamics measurement needs, from which orbits are determined, are summarized in Table 1.

A receiving antenna array with interferometry capability is also located at SAAMER-C (Figure 3). The array is a typical configuration for meteor radar systems consisting of five antennas, each of which is a three-element crossed yagi (Hocking et al. 1997). The receiving array is separated from the 


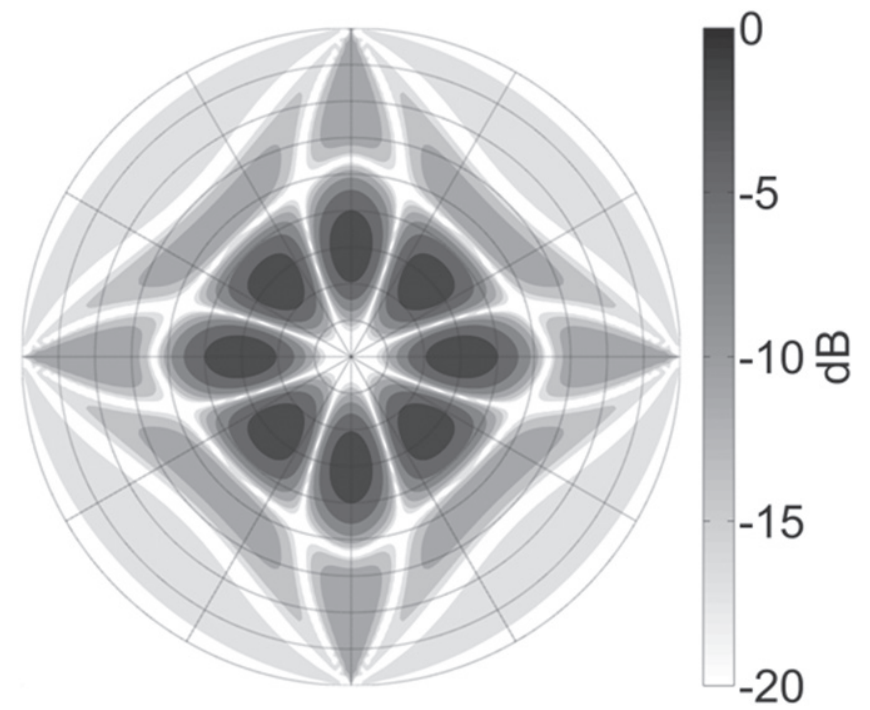

Figure 2. SAAMER transmit gain pattern in normal operation mode.

Table 1

SAAMER System Parameters

\begin{tabular}{lc}
\hline \hline Parameter & Value \\
\hline Peak transmitted power & $60 \mathrm{~kW}$ \\
Transmitting frequency & $32.55 \mathrm{MHz}$ \\
Pulse repetition frequency (PRF) & $1765 \mathrm{~Hz}$ \\
Bandwidth & $0.3 \mathrm{MHz}$ \\
Pulse width & $4 \mathrm{~km}$ \\
Range resolution & $2 \mathrm{~km}$ \\
Pulse code & 2 bit Barker \\
\hline
\end{tabular}

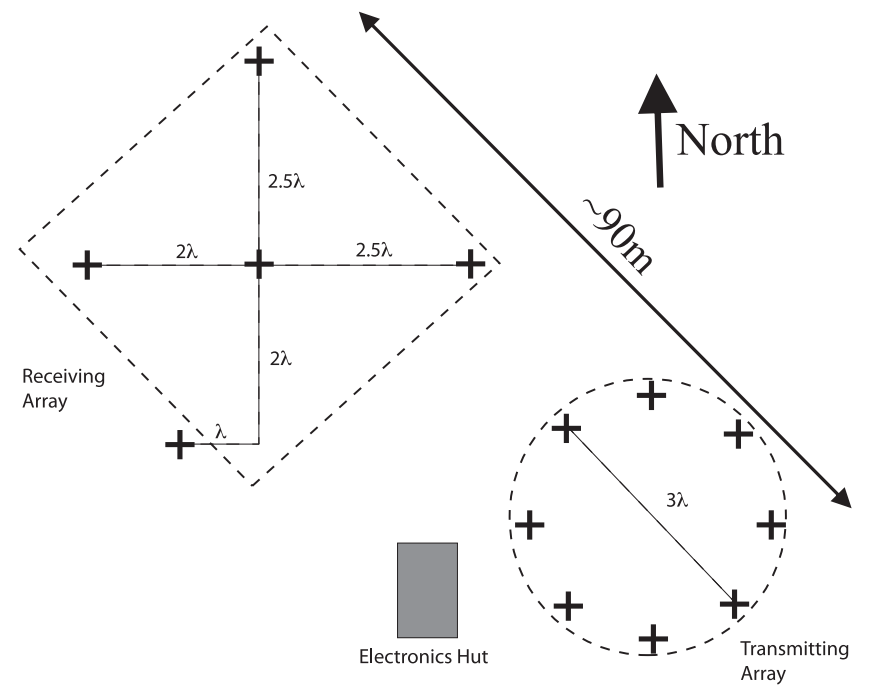

Figure 3. Antenna transmitter and receiver layout at Rio Grande, Tierra del Fuego (individual antennas are indicated with plus signs).

transmitting array by approximately $90 \mathrm{~m}$, which is several orders of magnitude less than the ranges at which meteors occur ( 100-400 km; Fritts et al. 2010b; Janches et al. 2014a). Thus, for the purposes of meteor observations, the SAAMER-C transmitting and receiving arrays can be treated as collocated.

The two remote stations, SAAMER-N and SAAMER-W, were deployed in 2010 August with the purpose of enabling meteoroid orbit determination and are each equipped with a single three-element crossed yagi receiving antenna. The remote stations were placed in such a way that they are in nearly orthogonal directions from SAAMER-C at a distance of the order of $10 \mathrm{~km}$. Instantaneously to the detection of a meteor by all three of the SAAMER-OS stations, the meteoroid trajectory and speed can be determined using the measured time delays between the detections (Baggaley et al. 1994; Webster et al. 2004; Brown et al. 2008). The meteoroid's orbit can be estimated using the procedure discussed in Section 2.2.

\subsection{Orbit Determination}

SAAMER uses the real-time meteor echo detection and analysis algorithms developed by Hocking et al. (2001) for SKiYMET systems. These algorithms simultaneously stream raw data into memory, detect meteor occurrences, and identify events produced by underdense specular trail reflections for separate storage. Meteor specular trails are semi-stationary targets, which drift primarily with the background wind at speeds typically less than $100 \mathrm{~m} \mathrm{~s}^{-1}$. Thus, when analyzing raw data, these meteor detections are found at the same range gate over many radar pulses until the trail diffuses in the atmosphere and the returned signal strength falls below the radar sensitivity threshold (Lau et al. 2006). In order to mitigate the occurrence of false meteor events, the system is typically configured to only consider as meteor events those that occur at the same range gate over a specified minimum number of consecutive transmit pulses. For SAAMER, this value is set to a minimum of four consecutive detections at the same range gate to be considered a meteor event. When a meteor is detected at SAAMER-C, the location (i.e., range, azimuth, and elevation) of the specular reflection point on the meteor trail is determined using the interferometric receiving array (Hocking et al. 2001; Lau et al. 2006). The specular reflection point (i.e., $t_{0}$ ) is defined as the point on the trail that minimizes the signal path from the transmit array to the meteor trail and then to the receiving antenna (McKinley 1961) and represents the location of the meteoroid at the time of detection (Hocking et al. 1997). Since the transmitting and receiveing arrays are treated as collocated at SAAMER-C, the range of the meteoroid is simply half of the measured signal path, and the specular reflection point is the point at which the meteoroid is at its minimum distance from the central station; the meteoroid's velocity is normal to the position vector from the radar. For the purposes of meteoroid orbit determination, the range, $\rho$, elevation, $\alpha$, and azimuth, $\phi$, give the position of the meteoroid relative to the central station in the local ENU frame (i.e., east, north, and up components), and thus

$$
\boldsymbol{r}_{\mathrm{ENU}}=\rho(\cos \alpha \sin \phi \hat{x}+\cos \alpha \cos \phi \hat{y}+\sin \alpha \hat{z}) .
$$

The meteoroid's absolute velocity is obtained with the remote sites using a geometrical technique similar to that employed at AMOR (Baggaley et al. 1994) and CMOR (Webster et al. 2004; Jones et al. 2005). This geometrical method is applied to all meteors that are detected at all three SAAMER stations and relies on the assumption that the interaction between the radar signal and the meteor trail is described by the specular reflection condition. This constrains the possible locations of the echo points for the remote station detections, which are from the forward scatter of the signal transmitted by SAAMER-C at different points along the meteor trail. The measured time delay between each of the detections 
at the remote sites and the central station allows for the determination of the meteoroid velocity (Baggaley et al. 1994; Brown et al. 2008), given by (Jones et al. 2005)

$$
\frac{d_{S} \cos \zeta}{v \Delta t_{S}}=\frac{2 \rho-d_{S} \sin \zeta}{\rho} \approx 2
$$

where $d_{S}$ is the distance of the remote station from the central station (i.e., $\rho>>d_{S}$ ), $v$ is the meteoroid speed, $\Delta t_{S}$ is the time delay between the detections at the remote and central stations, and $\zeta$ is the angle between the meteoroid velocity direction, $\hat{v}_{\text {ENU }}$, and the position vector of the remote station relative to the central station, $\boldsymbol{r}_{s}$, such that $\boldsymbol{r}_{s} \cdot \hat{v}_{\mathrm{ENU}}=d_{S} \cos \zeta$. Given the fact that the velocity direction is normal to the position from the central station (i.e., $\boldsymbol{r}_{\mathrm{ENU}} \cdot \hat{v}_{\mathrm{ENU}}=0$ ), the meteoroid trajectory is given by

$$
\left(\begin{array}{c}
\boldsymbol{r}_{\mathrm{ENU}} \\
\boldsymbol{r}_{\mathrm{SN}}^{T} \\
\boldsymbol{r}_{\mathrm{SW}}^{T}
\end{array}\right)=\left(\begin{array}{c}
0 \\
\Delta t_{N} \\
\Delta t_{W}
\end{array}\right) \boldsymbol{v}_{\mathrm{ENU}}
$$

where $\boldsymbol{r}_{\mathrm{SN}}$ and $\boldsymbol{r}_{\mathrm{SW}}$ are the position vectors of SAAMER-N and SAAMER-W with respect to SAAMER-C, respectively, and $\Delta t_{N}$ and $\Delta t_{W}$ are the time delays in the detection at the two remote sites relative to the detection time at the central site.

Given the position, velocity, and observation time of a meteoroid relative to an observer (e.g., a radar antenna), there are several methodologies that can be utilized to determine the meteoroid's heliocentric orbit (Jenniskens et al. 2011). For SAAMER-OS, a patched-conics approach (a method to simplify trajectory calculations for spacecraft in a multiplebody environment) is employed, as described by Wiesel (1997), where the meteoroid's geocentric and heliocentric orbits are considered separately and two distinct meteoroid orbit trajectories are determined: (1) the hyperbolic orbit about the Earth, and (2) the typically elliptical orbit about the Sun. For each of the two orbits, the center body (i.e., the Earth or the Sun) is considered to provide the dominant force acting on the meteoroid and is determined by the distance of the meteoroid from the relevant celestial bodies, as well as their mass properties. A barycentric Earth (i.e., Moon-Earth system) is utilized. All other forces (e.g., atmospheric drag) are modeled as perturbation forces and are not considered in the estimation of the meteoroid's nominal orbit. Mathematical details of coordinate conversion and orbit determination can be found in Wiesel (1997) and Bate et al. (1971).

\section{RADAR RESPONSE FUNCTION}

Ground-based meteoroid observations have several inherent biases that precondition what portion of the incoming flux can be detected by the particular technique and thus affect the measured distributions of meteoroid properties (Ceplecha et al. 1998; Galligan \& Baggaley 2004, 2005; Close et al. 2007; Janches et al. 2014b). For the case of radar observations, the biases are due to numerous factors, such as the meteor radar scattering mechanism (i.e., specular trail or head echo detection), the meteoroid interaction with the atmosphere, and the radar system parameters. In this work we refer to the system's performance with respect to local biases associated with the radar operating parameters and atmosphere effects as the radar response function (RRF; Ceplecha et al. 1998). The purpose of corrections via the
RRF is to account for in the final results the portion of incoming meteoroids that were not detected by the radar as a result of these effects (Galligan \& Baggaley 2004).

For SAAMER-OS, the RRF is estimated utilizing the methodology described by Equations (111) and (112) in Ceplecha et al. (1998) and carrying out the numerical integration for every position of the echo plane defined by a grid of radiant points ( $\alpha_{r}$ and $\phi_{r}$, where $r$ is the radiant). This method models the probability of the radar system to detect meteors produced by meteoroids with entry speed, $v_{\infty}$, based on the position of the meteoroid radiant as seen by an observer at the radar location, given in terms of the radiant azimuth, $\phi_{\infty}$, and elevation, $\alpha_{\infty}$. The RRF for a given radiant direction, after some algebra, is defined as

$$
\begin{aligned}
n_{r}\left(\phi_{\infty}, \alpha_{\infty}\right)= & A \sin ^{c} \alpha_{\infty} \int_{-\frac{\pi}{2}}^{\frac{\pi}{2}} \int_{h_{\min }}^{h_{\max }} \\
& \times\left(\eta(h) Q(h) \sqrt{G_{T} G_{R}}\right)^{c} \rho^{1-\frac{3 c}{2}} \frac{\partial \rho}{\partial h} d h d \phi
\end{aligned}
$$

where $\phi$ is the meteor echo plane azimuth defined in terms of the echo point elevation, $\alpha$, by

$$
\sin \alpha=\cos \alpha_{\infty} \cos \phi
$$

$h$ is the echo point altitude, $\rho$ is the meteor range, $G_{T}$ and $G_{R}$ are the radar transmit and receive antenna gain powers, $\eta$ is the fractional reduction in the meteor echo amplitude due to atmospheric attenuation factors, and $A$ is a constant coefficient that is derived from the radar signal wavelength, transmitted power, and minimum receivable power and may be neglected since it will scale the radar response equally for each radiant azimuth and elevation (Galligan \& Baggaley 2004). For the case of SAAMER, the limits in the integral in Equation (4), $h_{\text {min }}$ and $h_{\text {max }}$, are 70 and $110 \mathrm{~km}$, respectively, the altitude range where meteors are detected (Fritts et al. 2010b).

The cumulative mass index, $c$, in Equation (4) describes the mass distribution of the incoming meteoroids, where it is generally assumed that the incoming flux of meteoroids with masses greater than a given mass, $m_{0}$, is well represented by an inverse power law $\left(N\left(m \geqslant m_{0}\right) \propto m_{0}^{c}\right.$; Ceplecha et al. 1998, Equation (109)). The index value is assumed to be between 0.7 and 1.3. We adopt a value of 1.0 in this work, similar to that for the analysis of AMOR's observations (Galligan \& Baggaley 2004, 2005).

The modeled trail ionization profile function, $Q(h)$, represents the variation in electron line density with altitude within the meteor trail. For the case of underdense trails, where the electron density in the trail is sufficiently small so that secondary scatter between the electrons may be neglected, this function can be approximated as a Gaussian distribution and is given by (see discussion surrounding Equation (95) in Ceplecha et al. 1998)

$$
Q(h)=e^{-\frac{\left(h-h_{q}\right)^{2}}{2 \sigma_{Q}^{2}}}
$$

where $\sigma_{Q}$ gives the standard deviation of $Q$ with respect to altitude. The values of $h_{q}$ and $\sigma_{Q}$ are functions of the meteoroid entry speed and are given by Ceplecha et al. (1998) for several incoming speeds.

Finally, the fractional reduction in the meteor echo amplitude of the meteor echo return, $\eta$, is the product of four atmospheric 
factors. According to Ceplecha et al. (1998), these are (1) the finite initial radius of the trail, $\alpha_{r}$; (2) the rate of diffusion during trail formation, $\alpha_{v} ;(3)$ the decay rate of an established trail, $\alpha_{p}$; and (4) the Faraday rotation of the radar wave polarization plane, $\alpha_{f}$, such that $\eta=\alpha_{r} \alpha_{v} \alpha_{p} \alpha_{f}$. A value of 0 for the factor indicates that the signal is fully attenuated, while a value of 1 means that there is no attenuation. The details of the derivation of these attenuation factors, which are adopted in this work and, for reference, listed below, have been reported by many authors (Peregudov 1958; Ceplecha et al. 1998; Galligan \& Baggaley 2004).

The finite initial radius of the trail, $\alpha_{r}$, corrects for the fact that a large portion of the incoming meteoroid population is unobserved by ground-based radar because they create meteors at a height where the initial trail radius significantly attenuates the radar signal (Baggaley 1980; Steel \& Elford 1991) and is given by

$$
\begin{gathered}
\alpha_{r}=e^{-k^{2} r_{0}^{2}} \\
r_{0}=\left(6.0 \times 10^{3}\right) \delta_{0}^{-0.25} v_{\infty}^{0.6}
\end{gathered}
$$

where $r_{0}$ is the initial trail radius of the meteor trail, $k$ is the radar wavenumber, $v_{\infty}$ is the meteoroid velocity at the top of the atmosphere, and $\delta_{0}$ is the neutral number density.

The finite velocity attenuation factor, $\alpha_{v}$, takes into account the fact that meteor trails undergo diffusion within the atmosphere (Ceplecha et al. 1998). The rate of diffusion increases rapidly at higher altitudes owing to the exponential decrease of pressure with height (Galligan \& Baggaley 2004). If the rate of diffusion is high enough, there may be a significant reduction in the trail cross section prior to the formation of the central Fresnel zone (i.e., the specular reflection point, $t_{0}$ ), and the meteor trail may be completely undetected. The finite velocity factor is given by (Peregudov 1958)

$$
\begin{gathered}
\alpha_{v}=\frac{1-e^{-\Delta}}{\Delta} \\
\Delta=C_{\Delta} \frac{2 k^{2} D_{a} \sqrt{2 \rho \lambda}}{v_{\infty}}
\end{gathered}
$$

where $C_{\Delta}$ is 1.18 (Ceplecha et al. 1998) and $D_{a}$ is the ambipolar diffusion coefficient determined using the NRLMSISE-00 model from the US Naval Research Laboratory (Picone et al. 2002).

The pulse repetition rate attenuation factor, $\alpha_{p}$, accounts for the fact that, in some cases, the trail decay time is of the order of the radar inter-pulse period (IPP). If that happens, the trail will not be detected because it forms and decays between transmitted radar pulses (Ceplecha et al. 1998). Similarly, if the trail is required to be observed over $n$ consecutive transmit pulses, diffusion may cause the trail to decay before all of the pulses have been transmitted. This factor is given by

$$
\begin{gathered}
\alpha_{p}=\frac{1-e^{-w}}{w e^{w(n-1)}} \\
w=\frac{4 k^{2} D_{a}}{\mathrm{PRF}}
\end{gathered}
$$

where $\mathrm{PRF}=1 / \mathrm{IPP}$ is the radar pulse repetition frequency in $\mathrm{Hz}$ (Table 1). For the case of SAAMER-OS, this effect provides very little attenuation for meteors below $\sim 100 \mathrm{~km}$ altitude.

The Faraday rotation attenuation factor, $\alpha_{f}$, provides the last attenuation factor and is due to the rotation of the plane of polarization of the linearly polarized transmitted radar wave as it propagates through the ionosphere. This effect is expressed as (Galligan \& Baggaley 2004)

$$
\begin{gathered}
\alpha_{f}=\cos 2 \Omega_{f} \\
\Omega_{f}=\left(2.36 \times 10^{4}\right) f^{-2} \int B \cos \chi q(s) d s
\end{gathered}
$$

where $\Omega_{f}$ is the angle of rotation, $f$ is the radar operating frequency in $\mathrm{Hz}, B$ is the local geomagnetic field in $\mathrm{Wb} \mathrm{m}^{-2}, \chi$ is the angle between the magnetic field and the wave propagation direction, and $q(s)$ is the electron line density per cubic meters along the path element $d s$. Galligan \& Baggaley (2004) found that the Faraday rotation effect is small enough at low altitudes so that it may be neglected, and thus the value of $\alpha_{f}=1.0$ is adopted in this study.

The RRF is determined for each observed meteor to estimate the detection probability of a meteoroid with a given entry speed, radiant, and observed time. This probability is then used as a weighting factor, $w_{r}=n_{r}^{-1}$, to correct the observations for the effects described above, where $n_{r}$ is derived from Equation (4). Essentially, $w_{r}$ scales each of the observed meteoroid distributions (i.e., entry speed, radiant, and the orbital parameters) by assuming that for each observed meteor there are $w_{r}$ meteors with the same parameters that were not detected by the radar (Galligan \& Baggaley 2004, 2005). It is important to note that the RRF corrects for meteoroids that produce meteors with trail electron line density above the sensitivity threshold of the radar, and thus the results are equivalent to the distributions weighted for a limiting trail line density. If a population of particles with velocities too low and/or masses too small to produce sufficient ionization exists and is completely undetected by the radar, as argued by Nesvorný et al. (2010, 2011a), the RRF will not take into account such effect.

\subsection{Normalization Factors}

As discussed in the previous section, the RRF takes into account the sensitivity threshold of the instrument to detect a meteor trail electron line density. Previous meteoroid surveys, however, have reported their results weighted with additional factors, such as the limiting meteoroid mass (Taylor \& Elford 1998; Campbell-Brown 2008). The trail line density is dependent on the meteoroid mass and speed, and its relationship is referred to as the meteoroid ionization efficiency and is given by (Bronshten 1983)

$$
q \propto m v^{3.42} .
$$

This implies that meteoroids with higher speeds are much more likely to be observed than slower ones, and so the smallest detectable mass for a given radar system decreases with increasing speed. ${ }^{10}$ This allows for the limiting mass

\footnotetext{
${ }^{10}$ Several authors have revisited this equation and in some cases have revised it. See, for example, the discussion concerning Equation (43) in Ceplecha et al. (1998), Section 2.1 in Weryk \& Brown (2013), and Section 5.2 in Janches et al. (2014b). The exponent in the velocity of Equation (15) can be anywhere between 3 and 4 . We chose 3.42 for this work because the previous surveys to which we are comparing our results used similar values (Brown et al. 2004; Galligan \& Baggaley 2005).
} 
distribution to be found from the radar-response-corrected results by applying a mass weighting factor, $w_{m}$, given by

$$
w_{m}=\left(\frac{v}{v_{\text {ref }}}\right)^{-3.42}
$$

where $v_{\text {ref }}$ is a reference speed to make the weighting factor unitless. For SAAMER-OS, we adopt, for convenience, $v_{\text {ref }}$ as $1 \mathrm{~km} \mathrm{~s}^{-1}$, and applying this weighting to the observed distributions results in the distributions of meteoroids above the smallest detectable mass at $v_{\text {ref. }}$.

In this work we will explore also an additional distribution weighting factor introduced by a limiting meteoroid kinetic energy, which is particularly useful for the purposes of characterizing the threat of meteoroid collisions with spacecraft (Campbell-Brown 2008). Similar to the limiting mass distributions, the distributions weighted for limiting kinetic energy are found by applying a weighting factor, $w_{k e}$, to the radar response corrections given by

$$
w_{\mathrm{ke}}=w_{m}\left(\frac{v}{v_{\mathrm{ref}}}\right)^{2}=\left(\frac{v}{v_{\mathrm{ref}}}\right)^{-1.42} .
$$

\section{RESULTS}

In this section, the results obtained between 2012 January 21, when SAAMER-OS orbital determination became operational, and 2015 April are presented. In addition and when available, the SAAMER observations are compared with those obtained by previous surveys. In particular, in Section 4.1, the raw distribution without the application of any correcting or weighting factor is presented, while Section 4.2 presents the results corrected by the RRF weighted for both the limiting mass and kinetic energy factors.

\subsection{Observed Results}

SAAMER-C detects between 15,000 and 25,000 meteor events daily (Fritts et al. 2010b; Janches et al. 2014a); however, in order to determine a meteoroid's orbital properties, the meteor must be observed by all three of the SAAMER-OS stations. This results in about an order of magnitude less detections than those recorded by SAAMER-C alone. Figure 4 shows the daily count of determined meteoroid orbits observed throughout the surveyed period (2012 January-2015 April), where it can be seen that the system can measure up to $\sim 1800$ meteoroid orbits per day. Unfortunately, SAAMER-OS performance, from 2012 December to 2013 July (arrow labeled $\mathrm{T} 1$ in panel (a) and arrow labeled T2 in panel (b) of Figure 4, respectively), suffered from a failure in the transmit power amplifiers. Operations resume immediately after the repair of these amplifiers; however, the number of recorded orbits was somewhat lower than in the previous year owing to an external source of noise that could not be mitigated until approximately a year later (arrow labeled T3 in panel (c)). Despite this interruption, a total of 793,577 meteoroid orbits were measured as of 2015 April 30 by SAAMER-OS. This statistic is larger than that recorded by AMOR over a 5-year period (Galligan \& Baggaley 2004, 2005). Therefore, the SAAMER-OS campaign is the second-largest orbital data set overall, after CMOR's, and also the largest in the southern hemisphere.

Figure 5 shows the distribution of the observed meteoroid geocentric speeds (i.e., relative to the radar location)
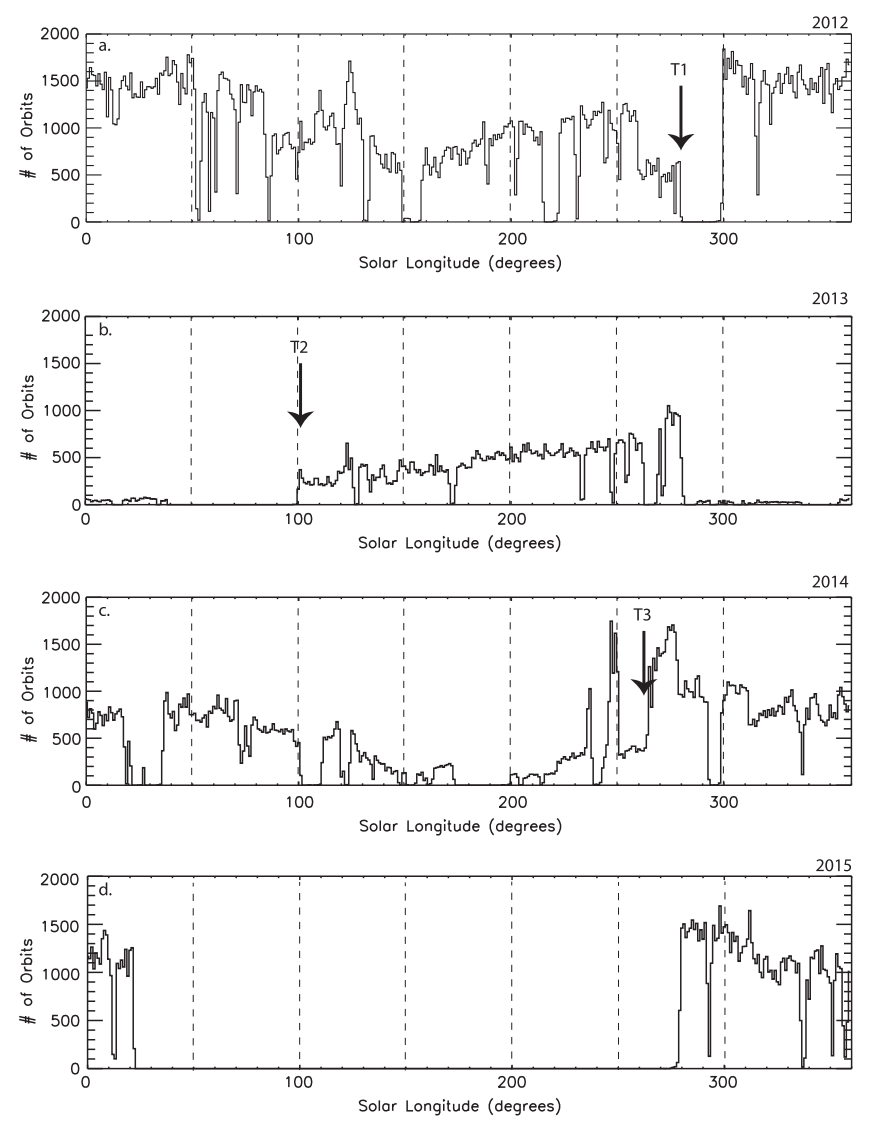

Figure 4. Daily count of meteoroid orbits as a function of solar longitude measured by SAAMER-OS since 2012 . The bin size is $1^{\circ}$ in solar longitude.

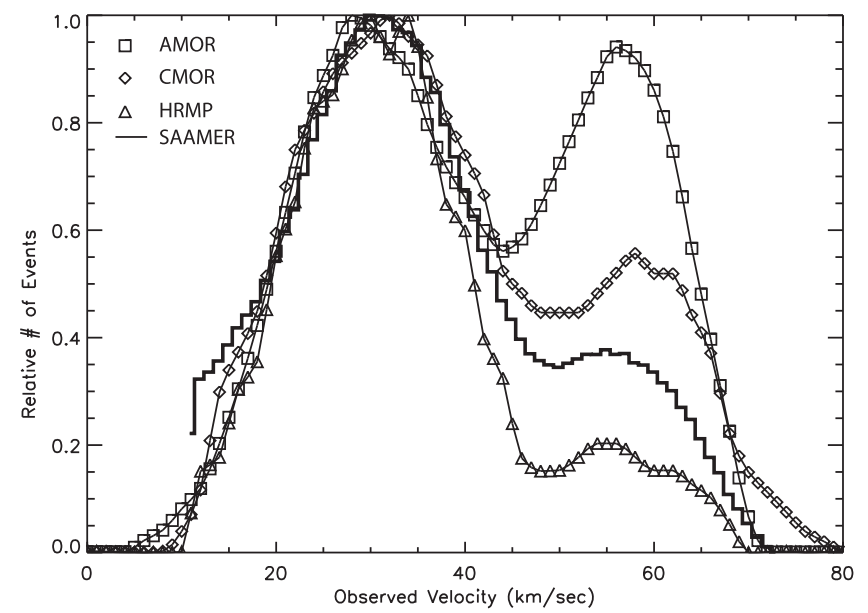

Figure 5. Observed meteoroid speed distributions for SAAMER-OS, AMOR, CMOR, and HRMP. The bin size is $1 \mathrm{~km} \mathrm{~s}^{-1}$ in velocity.

determined by SAAMER-OS, as well as the same distributions by AMOR (Galligan \& Baggaley 2004), CMOR (CampbellBrown 2008), and HRMP (Jones \& Brown 1993). SAAMEROS's speed distribution has a bimodal shape with a dominant peak at approximately $30 \mathrm{~km} \mathrm{~s}^{-1}$ and a weaker peak at $\sim 55 \mathrm{~km} \mathrm{~s}^{-1}$. The bimodal nature of the distribution is also present in the previous surveys. For the dominant and slower speed there is a general agreement between the SAAMER-OS results and those obtained by previous surveys. The agreement is not only on the center velocity but also on the distribution 


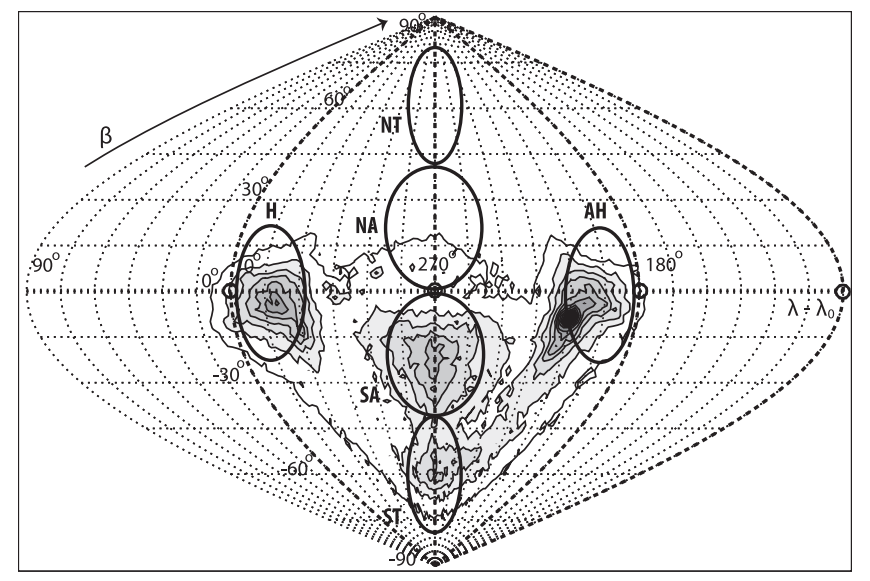

Figure 6. Observed meteoroid radiant ecliptic latitude and longitude by SAAMER-OS. The bin size is $2^{\circ}$ for both coordinates.

width. The high-speed distribution, however, appears to have a higher intensity, relative to the dominant peak, in the AMOR and CMOR results and lower intensity in the HRMP results. This is largely due to the fact that the initial trail radius attenuation decreases with increasing radar operating frequency (Equation (7)), mainly affecting the detections of high-speed meteoroids. The operating frequencies for AMOR, CMOR, SAAMER, and HRMP are 26, 29, 32, and $41 \mathrm{MHz}$, respectively, which is consistent with the fact that the least attenuation is seen in AMOR observations and the strongest is observed in the HRMP results.

The distribution of the observed meteoroid radiants is shown in Figure 6, which represent the points in the sky where the meteoroid entered into a hyperbolic geocentric orbit (Jones \& Brown 1993). The radiants are displayed in ecliptic coordinates in which they are viewed from an Earth-centered frame of reference: the radiants ${ }^{11}$ in the plot are a function of $\lambda-\lambda_{0}\left(\lambda_{0}\right.$ is the true longitude of the Sun); therefore, the heliocentric motion of the Earth is effectively removed and the position of each source fixed in ecliptic coordinates throughout the year is displayed (e.g., the Earth's apex is always at $270^{\circ}$ ). Figure 6 is oriented such that the center point corresponds to the apex direction $\left(\beta=0, \lambda-\lambda_{0}=270^{\circ}\right)$ and the helion $\left(\beta=0, \lambda-\lambda_{0}=\right.$ $0^{\circ}$ ) is to the left of the apex. For reference, the locations of the six sporadic meteoroid apparent sources are also represented as ellipses in the figure, with the coordinates as specified in Fentzke \& Janches (2008). These are the north and south apex $(\mathrm{N} / \mathrm{SA})$, the north and south toroidal (N/ST), and the helion (H) and antihelion (AH). Contributions from the sporadic apparent sources to the radiant distribution observed by SAAMER-OS are evident in this figure, as well as two strong meteor showers, which appear as dense concentrated enhancements in the radiant distribution (Janches et al. 2014a). The strong enhancement within the AH source $\left(\beta \sim-10^{\circ}, \lambda-\lambda_{0} \sim\right.$ $210^{\circ}$ ) corresponds to the Southern $\delta$ Aquarids (SDA) shower, whose activity is so strong that it dominates the color scale of Figure 6. The weaker enhancement to the left of the NA source ( $\beta \sim 5^{\circ}, \lambda-\lambda_{0} \sim 294^{\circ}$ ) corresponds to the $\eta$ Aquarids shower (ETA; Brown et al. 2010). As expected, the majority of meteors observed by SAAMER-OS originate from radiant locations south of the ecliptic (i.e., the ecliptic latitude is negative), with

${ }^{11}$ Usually displayed as a function of the ecliptic longitude, $\lambda$.
Table 2

Observed Sporadic Apparent Source Contributions

\begin{tabular}{lcccr}
\hline \hline Source Name & \multicolumn{4}{c}{ Contribution (\%) } \\
\cline { 2 - 5 } & SAAMER-OS & AMOR & CMOR & HRMP \\
\hline North Apex & 5.5 & 52 & 21 & 17.6 \\
South Apex & 29.4 & & 10 & 6.0 \\
Helion & 20.8 & 16 & 22 & 11.8 \\
Anti-helion & 25.6 & 27 & 29 & 20.4 \\
North toroidal & 0.3 & $\ldots$ & 18 & 44.2 \\
South toroidal & 17.3 & $\ldots$ & $\ldots$ & $\ldots$ \\
\hline
\end{tabular}

particularly strong contributions from the SA, ST, H, and AH sources.

The relative source strengths derived from the SAAMER-OS observations are summarized in Table 2 and compared with those derived from the AMOR, CMOR, and HRMP surveys (Jones \& Brown 1993; Campbell-Brown 2008). Note that the apex results from the AMOR survey are not separated between the southern and northern contributions, as they are presented in Galligan \& Baggaley (2005). Despite the strong initial trail radius effect that significantly reduces the detection of highspeed meteors, the SA source, which is characterized by highvelocity meteoroids (Fentzke \& Janches 2008) probably originating from Oort Cloud comets (Galligan \& Baggaley 2004; Nesvorný et al. 2011b), provides the strongest observed contribution in the SAAMER-OS observations; however, the $\mathrm{H}$ and $\mathrm{AH}$ sources collectively provide most of the meteoroids, resulting in the $30 \mathrm{~km} \mathrm{~s}^{-1}$ peak in the observed speed distribution. In addition, the ST source results via SAAMER-OS highlight this system's unique properties, and it is also a fresh subject on the "scientific market." AMOR and CMOR observed similar trends to each other; however, the apex sources were much stronger in the AMOR observations. This is most likely due to a lower initial trail radius attenuation given the radar's lower transmitted frequency. The observations by HRMP were at mid-northern latitude and experienced the largest attenuation from the initial trail radius effect. Consequently, meteoroids from the apex sources were largely undetected, and the NT presence was dominant in these observations (Jones \& Brown 1993).

It is interesting to note that the previous surveys reported an observed difference between the contributions of the $\mathrm{H}$ and $\mathrm{AH}$ sources, resulting in a stronger contribution by the latter. Although not as strong as the past surveys, a similar difference is observed in the SAAMER-OS results. The actual contributions from the $\mathrm{H}$ and $\mathrm{AH}$ sources are expected to be approximately equal as these sources both consist of meteoroids that have heliocentric orbits with high eccentricity and low inclination and are likely from Jupiter-family comets (JFCs; Taylor \& Elford 1998; Jenniskens 2006, 2008; Nesvorný et al. 2010, 2011a). The fact that the number of detections from the $\mathrm{AH}$ is higher may be the result of meteoroid destruction mechanisms during perihelion, as well as indicating possible observational biases associated with radar systems, as explained by Wiegert et al. (2009).

Finally, the distributions of the heliocentric orbital parameters of semimajor axis $(a)$, eccentricity $(e)$, and inclination (i) are given in Figure 7, where a general agreement among the different survey results is evident. Specifically, the semimajor axis distribution in the top panel shows that the majority of the meteoroids observed have $a$ values close to that of the Earth's 

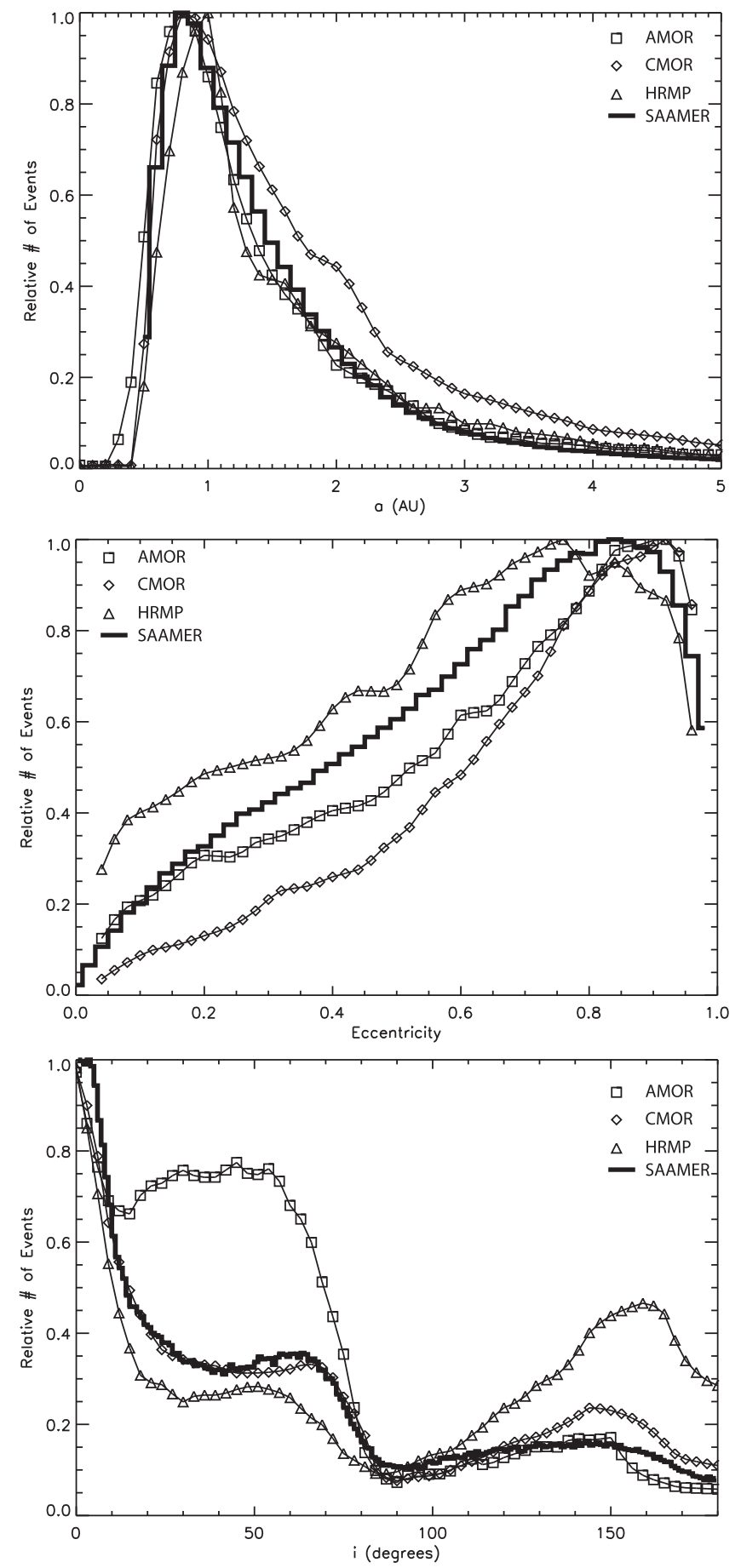

Figure 7. Observed meteoroid heliocentric orbital parameters by SAAMEROS, AMOR, CMOR, and HRMP. Top: $a$; middle: $e$; bottom: $i$. The bin size is $0.1 \mathrm{AU}, 0.02$, and $1^{\circ}$ for each of the respective quantities.

(i.e., $1 \mathrm{AU}$ ). The distribution of $e$ (middle panel) shows that the majority of observed meteoroids have large eccentricities, unlike Earth's near-circular orbit about the Sun. This is an expected result as most sporadic meteoroids likely originate from cometary parent sources, which themselves generally have high eccentricity (Jenniskens 2006, 2008; Wiegert et al. 2009; Nesvorný et al. 2010, 2011b, 2011a; Pokorný et al. 2014). Furthermore, this is also expected given the strong percentage of observed meteoroids that appear to originate from either the helion or anti-helion direction. The distribution of orbit inclination shows agreement among SAAMER-OS, AMOR, and CMOR observations for the case of meteoroids in prograde orbits (i.e., inclination less than $90^{\circ}$ ). On the other hand, for retrograde meteoroids (i.e., inclination greater than $90^{\circ}$ ), the SAAMER-OS observations are consistent with HRMP and CMOR. The larger percentage of retrograde meteoroids observed by AMOR can be attributed to the stronger contribution of apex meteoroids to the distribution due to the smaller attenuation effect of the initial trail radius (Campbell-Brown 2008). The estimate on the orbital elements is acceptable at an order of $5 \%-10 \%$ the given value, comparable to what is reported by Brown et al. (2004) for CMOR. We aim to develop in the near future a Monte Carlo model for the underdense echo uncertainty estimation, in order to explicitly give a better estimation of the standard deviation in the values.

\subsection{Radar-response-corrected Results}

The observed distributions presented in Section 4.1 show some differences among the observed results from the various surveys, particularly in the distributions of speed, radiant, and inclination. These differences show how the radar response biases, discussed in Section 3, affect each radar system differently. In order to obtain an accurate estimation of meteoroid properties, these biases must be corrected. In this section we correct the distributions observed by SAAMER-OS using the $w_{r}$ weighting factor. This results in distributions weighted for meteoroids that produce trails with ionization line density above the minimum detectable threshold for the given system. Additionally, $w_{m}$ and $w_{\mathrm{ke}}$ are also applied to determine the distributions for meteoroids above a limiting mass and kinetic energy threshold, respectively. Thus, the radarresponse-corrected results consist of three distributions weighted for different limiting factors (minimum trail line density, limiting mass, and limiting kinetic energy) and used in this work to compare the SAAMER-OS distributions with those from previous surveys.

\subsubsection{Corrected Velocity Distributions}

Figure 8 shows the geocentric meteoroid speeds corrected by the RRF and weighted by the three factors mentioned above. The importance of specifying the weighting factors used in the correction is clearly apparent in this comparison. For the minimum trail line density correction (i.e., RRF without any additional weighting), the distribution is bimodal with a dominant peak centered around $32 \mathrm{~km} \mathrm{~s}^{-1}$ and a secondary peak that has slightly shifted to higher speed compared to the uncorrected distribution. The relative numbers of faster meteors also increased owing to the attenuation experienced by SAAMER-OS caused by the initial trail radius effect. The radar response correction accounts for this effect, and thus the number of high-speed meteors is increased, as expected. On the other hand, weighting the distributions by the limiting mass distribution results in a sharp increase of meteoroids with velocities on the order of $11-15 \mathrm{~km} \mathrm{~s}^{-1}$ as these particles will produce, for a given mass, two orders of magnitude less ionization compared to those at high speeds (Bronshten 1983; Vondrak et al. 2008; Janches et al. 2014b). Note that the characteristics of the initially observed distribution are no longer evident and appear to be dominated by the velocity dependence on ionization efficiency defined by Equation (15). 


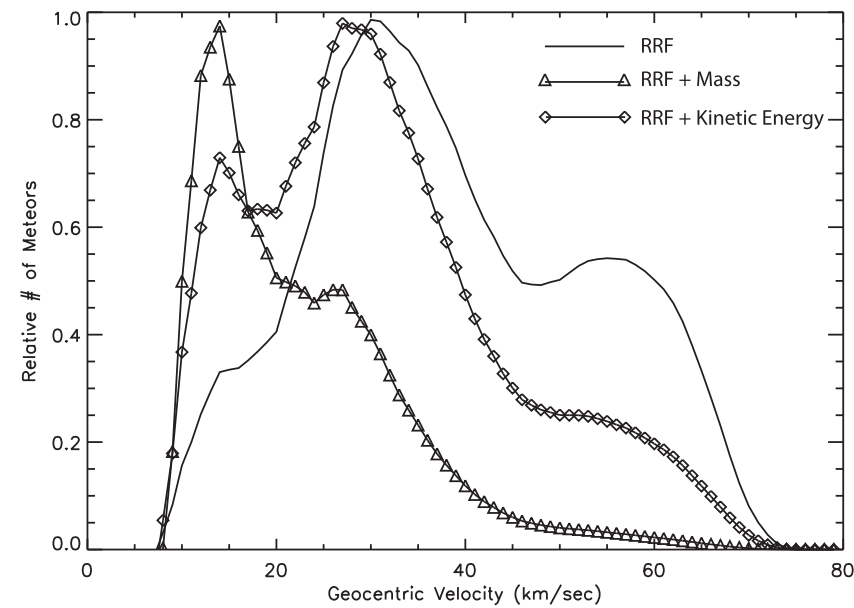

Figure 8. Radar response correction of meteoroid speed observed by SAAMER-OS weighted for limiting trail line density (black), limiting mass (triangle), and limiting kinetic energy (diamond). The bin size is $1 \mathrm{~km} \mathrm{~s}^{-1}$ in velocity.

This speed distribution is in closer agreement with interplanetary models of the ZDC (Drolshagen et al. 2008; Nesvorný et al. 2010). Finally, the radar-response-corrected distribution weighted for limiting kinetic energy provides a better balance between the directly observed trends and the expected trends regarding meteoroid size or mass. The primary and secondary peaks in the observed distributions are still evident in the corrections weighted for limiting kinetic energy but have shifted to a slightly slower speed. In addition, the sharp peak at very slow speeds evident in the limiting-mass-weighted distribution still remains and is of similar significance to the observed dominant peak at $25-30 \mathrm{~km} \mathrm{~s}^{-1}$. The three weighted speed distributions presented in Figure 8 show that the distribution of a given meteoroid property can vary significantly depending on the methodology (i.e., weighting factors) used, and it is essential to clearly specify and consider these factors in any discussion of the properties of sporadic meteoroids.

Regarding the comparison between the SAAMER-OS corrected results and those from previous surveys, only CampbellBrown (2008) and Taylor \& Elford (1998) reported corrected distributions for a constant limiting mass for CMOR and HRMP, respectively, and these are reproduced in Figure 9, together with the limiting-mass-weighted distributions provided by SAAMER-OS. Since Galligan \& Baggaley (2004) did not specify whether the results were weighted for trail line density, mass, or some other factor, we do not include AMOR's results in the comparison displayed in Figure 9. It can be seen from this comparison that SAAMER's results are in close agreement with those resulting from CMOR's observations for meteoroid velocities lower than $15 \mathrm{~km} \mathrm{~s}^{-1}$ or greater than $30 \mathrm{~km} \mathrm{~s}^{-1}$. SAAMER's distribution peaks at $\sim 14 \mathrm{~km} \mathrm{~s}^{-1}$, while CMOR's peak is centered only $1 \mathrm{~km} \mathrm{~s}^{-1}$ higher. For meteoroid velocities between 15 and $30 \mathrm{~km} \mathrm{~s}^{-1}$, however, CMOR distribution shows a larger presence of these meteors.

\subsubsection{Corrected Radiant Distributions}

The SAAMER-OS radar-response-corrected radiant distributions weighted for minimum detectable trail line density,

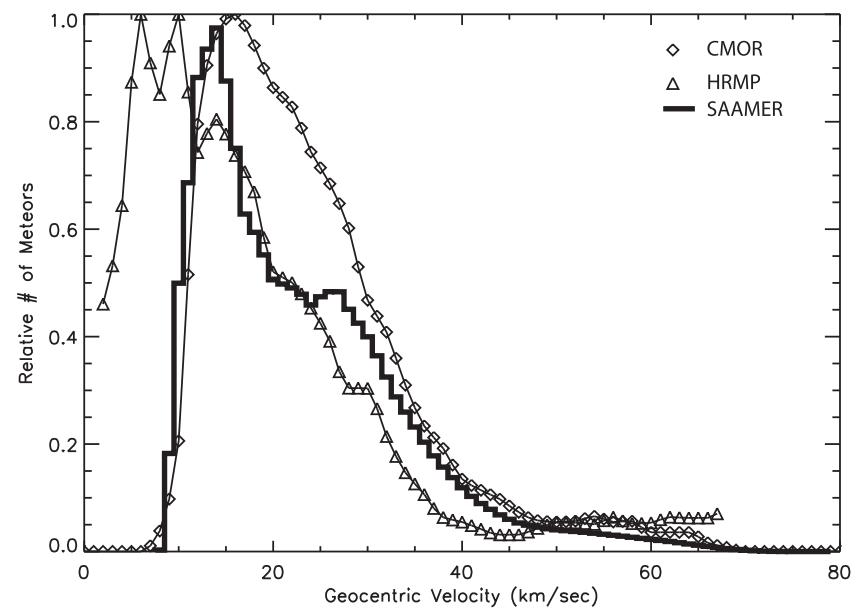

Figure 9. Radar-response-corrected meteoroid speed weighted for limiting mass by SAAMER-OS compared to reported distributions by CMOR and HRMP. The bin size is $1 \mathrm{~km} \mathrm{~s}^{-1}$ in velocity.

limiting mass, and limiting kinetic energy are given in Figure 10. The top panel of this figure shows that the activity from the SA source is enhanced by the RRF correction. In addition, the radiant enhancements are due to meteor showers becoming less intense as compared to the uncorrected distribution in Figure 6. Additionally, the contributions from the $\mathrm{H}, \mathrm{AH}$, and ST sources also increase as compared to those from the uncorrected distributions. In both the limiting-massand limiting-kinetic-energy-weighted distributions (middle and bottom panels), the $\mathrm{H}$ and $\mathrm{AH}$ become the dominant contributors, while the contribution of the SA is significantly reduced. The ST source is still apparent; however, its contribution compared to the limiting trail line density contribution appears to be reduced. These results are expected since meteoroids from the apex sources have faster speeds in comparison to the other sources, while the helion and antihelion sources contain a significant portion of slow meteoroids as the intensity of these sources increased significantly after weighting for mass (Fentzke \& Janches 2008).

The contributions weighted by the RRF of each sporadic source are given in Table 3 . The table provides the corrected values for SAAMER-OS, as well as reported corrected source strengths for AMOR (Galligan \& Baggaley 2005) and CMOR (Campbell-Brown 2008). The HRMP source contributions are not reported in the table because Taylor \& Elford (1998) did not present numerical values of the corrected source contributions. SAAMER-OS's results show an increased contribution from the south apex when the initial trail radius (i.e., RRF) effect is applied. The RRF reduces the probability of detection of high-speed meteoroids (Ceplecha et al. 1998). However, the apex contributions are ultimately reduced when considering the limiting-mass- or kinetic-energy-weighted distributions, as these limiting factors apply very small weighting factors to high-speed meteoroids. Also seen in Table 3 is that the contributions of the apex sources are also significantly reduced in both AMOR and CMOR corrected results. This is expected in particular in CMOR's results, given that it is calculated for a constant limiting mass. Furthermore, SAAMER-OS and CMOR results show similar overall contributions from the apex; however, SAAMER-OS observed a significant asymmetry between the north and south apex owing to its southern location $\left(53^{\circ} \mathrm{S}\right)$, limiting severely the visibility of the 

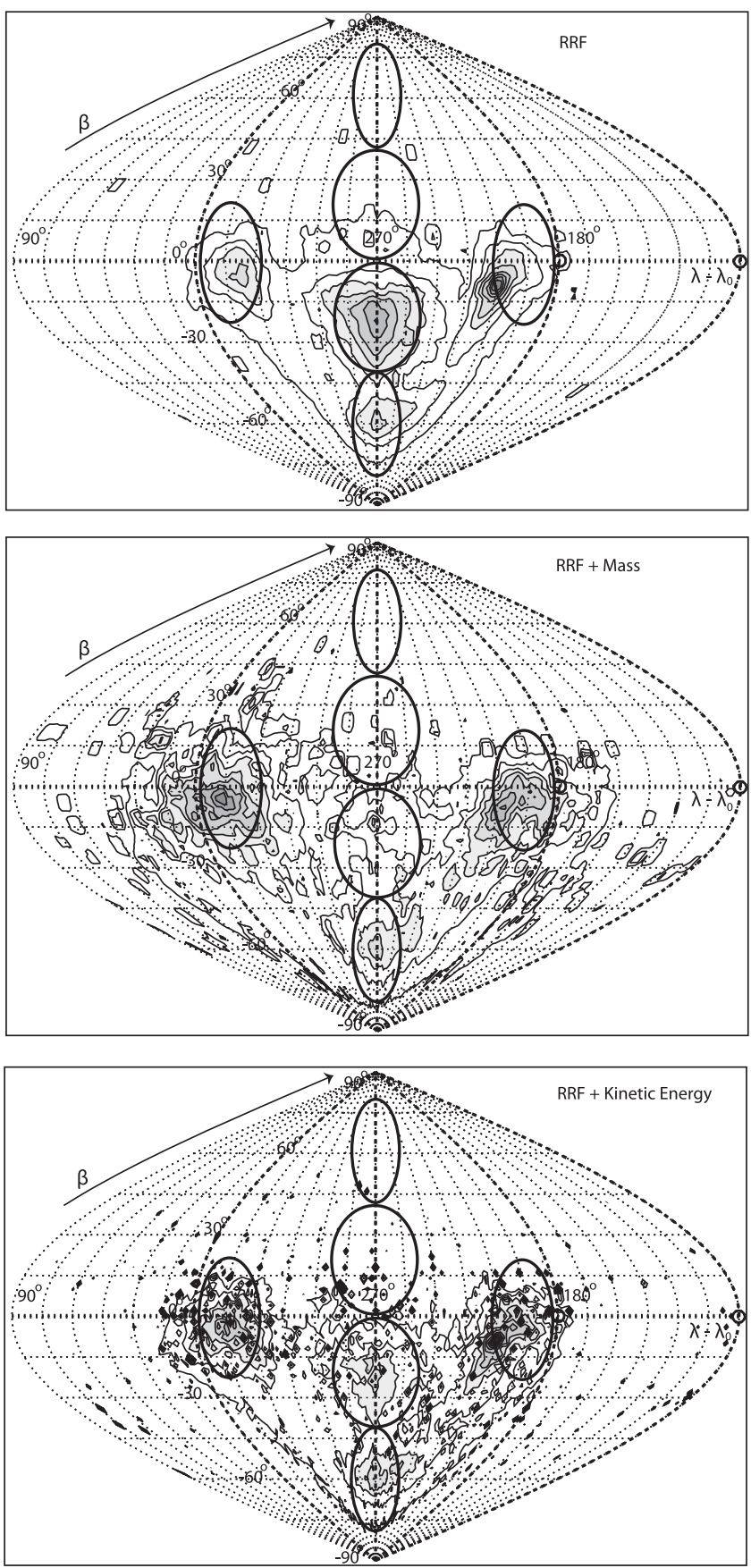

Figure 10. SAAMER-OS radar-response-corrected radiant distributions weighted for limiting trail line density (top), limiting mass (middle), and limiting kinetic energy (bottom). The bin size is $2^{\circ}$ for both coordinates.

north apex. In contrast, since CMOR is located at approximately $43^{\circ} .2 \mathrm{~N}$, its ability to observe the south apex source is relatively better than SAAMER-OS's ability to detect particles from its northern counterpart. The significant decrease in the apex meteoroids in the AMOR corrections is still puzzling, and since Galligan \& Baggaley (2005) do not discuss weighting the corrected distributions for a limiting mass, kinetic energy, or any other property, the causes of this decrease cannot be inferred. If the distribution reported by the authors is weighted only for the minimum detectable electron line density (i.e., the radar response correction is applied without any additional weighting factors), then it should resemble the observed
Table 3

Comparison of the SAAMER-OS RRF Corrected Sporadic Source Strengths Weighted for Trail Line Density $(q)$, Mass $(m)$, and Kinetic Energy (KE) with Those Derived from AMOR and CMOR

\begin{tabular}{|c|c|c|c|c|c|}
\hline \multirow{3}{*}{ Source Name } & \multicolumn{5}{|c|}{ Contribution (\%) } \\
\hline & \multicolumn{3}{|c|}{ SAAMER-OS } & \multirow{2}{*}{$\frac{\mathrm{AMOR}}{q}$} & \multirow{2}{*}{$\frac{\text { CMOR }}{m}$} \\
\hline & $q$ & $m$ & $\mathrm{KE}$ & & \\
\hline North apex & 6.1 & 4.5 & 4.3 & 23 & 11 \\
\hline South apex & 36.3 & 14.9 & 24.3 & & 11 \\
\hline Helion & 17.1 & 31.0 & 23.6 & 31 & 31 \\
\hline Anti-helion & 20.2 & 33.0 & 27.0 & 40 & 37 \\
\hline North toroidal & 0.2 & 0.8 & 0.3 & $\cdots$ & 10 \\
\hline South toroidal & 19.8 & 15.7 & 20.3 & $\ldots$ & $\ldots$ \\
\hline
\end{tabular}

distributions more closely, with perhaps an increase in the apex contributions due to the initial trail radius effect. The distribution resulting from the AMOR corrections, however, closely matches the distribution at CMOR, which suggests that the reported distribution may have been, in fact, weighted for a constant limiting mass. This emphasizes the importance of not only applying the bias corrections but also explicitly mentioning what the corrected distributions represent, so that accurate comparisons among different systems can be made.

The helion and anti-helion sources are dominant in the SAAMER-OS results for the distributions that are weighted for a limiting mass. Likewise, these sources are also dominant in the AMOR and CMOR results; however, these systems estimate a much stronger contribution by the anti-helion source than found with the SAAMER-OS corrected results. Taylor \& Elford (1998) found the same asymmetry between the helion and anti-helion sources in the corrected radiant distribution for HRMP. In contrast, the SAAMER-OS distribution shows an approximately equal contribution from the helion and antihelion sources. Taylor \& Elford (1998) attributed this asymmetry to significant trail attenuation from the Faraday rotation effect, which can be significant during the day under conditions of high solar activity, such as during a solar maximum. The SAAMER-OS observations, however, also took place during a solar maximum, and though the Faraday rotation effect was considered, there was not a significant asymmetry in the corrected distributions. More recently, Wiegert et al. (2009) suggest that the asymmetry is dynamical in nature and should be more significant for smaller meteoroid masses, while Nesvorný et al. (2010, 2011a) show that the dynamical evolution of JFC particles should result in two symmetrical sources. It is important to note that while Nesvorný et al. (2010, 2011a) utilized a large population of comets, resulting in a uniform distribution of longitudes of ascending nodes and periapsis, Wiegert et al. (2009) used only specific parent bodies.

Finally, a rather encouraging result is the ability of SAAMER-OS to observe a strong contribution from the south toroidal source, which has been evasive in all the previous surveys (Section 5). The significant contribution of the south toroidal meteoroids in the SAAMER-OS results is partially due to its far southern latitude; however, the AMOR system did not specifically report particles from this region despite being located at similar southern latitude $\left(\sim 43^{\circ} \mathrm{S}\right.$; Galligan \& Baggaley 2004) to that of CMOR's northern latitude. In addition, the intensity of this source appears to be stronger, 
regardless of the corrected distribution utilized, than the northern counterpart, as evident in CMOR's observations. These results may suggest an asymmetry between the overall global contributions of the north and south toroidal meteoroids and are in contrast with the results by Pokorný et al. (2014), who suggest that Halley-type comets should contribute equally to the formation of these sources. This emphasizes the importance of continuous measurements using SAAMER-OS given its unique ability to observe this poorly studied sporadic meteor source and to confirm this possible evidence of different contributions from north and south toroidal meteoroids.

\subsubsection{Corrected Orbital Element Distributions}

The distributions for heliocentric semimajor axis, eccentricity, and inclination resulting from the SAAMER-OS radar response correction and weighted for limiting trail line density, mass, and kinetic energy are given in Figure 11. Looking at the top panel of this figure, it can be seen that there is general agreement among the semimajor axis distributions, with a dominant peak at approximately $1 \mathrm{AU}$ for all of the weighted distributions. However, it can also be observed that the limiting mass distribution shows fewer meteoroids with large semimajor axis, suggesting that for a given mass, there are more meteoroids entering Earth's atmosphere that are in heliocentric orbits confined to the inner solar system. This implies that these meteoroids are from either asteroidal parent sources or cometary parents with significantly decayed orbits owing to PR drag (i.e., they were ejected from their parents relatively long ago; Wiegert et al. 2009; Nesvorný et al. 2010, 2011a).

The SAAMER-OS corrected eccentricity distributions are displayed in the middle panel of Figure 11. The eccentricity distributions weighted for trail line density and kinetic energy both show a dominance of meteoroids with highly eccentric orbits, consistent with the hypothesis that most of the SMC is composed of cometary sources (Wiegert et al. 2009; Nesvorný et al. 2010, 2011a, 2011b; Pokorný et al. 2014). The limiting mass distribution, however, shows a drastically different trend than the uncorrected observed distribution, where the majority of meteoroids have much lower eccentricity. Moreover, these meteoroids are likely to have slow speed and originate from the helion or anti-helion sources, as suggested by the results presented in this section, and are consistent with the ZDC model developed by Nesvorný et al. (2010, 2011a), which suggests that a significant percentage of helion and anti-helion meteoroids have small semimajor axis, low eccentricity, and slow speed.

The bottom panel of Figure 11 shows the effect that the different weightings have on the SAAMER-OS inclination distribution, where a dominance of low-inclination, prograde orbits representative of the helion and anti-helion sources is present. When considering the results weighted by the minimum detectable trail line density effect, a large percentage of retrograde meteoroids with high inclination and also prograde orbits become evident. These originate from the apex and south toroidal meteoroid sources, respectively. When additionally weighting for a limiting mass and kinetic energy, however, the contribution of retrograde meteoroids is reduced. This is consistent with the overall reduction of the apex and south toroidal meteoroids evident in the radiant distributions displayed in Figure 10. Additionally, the large percentage of meteoroids with small eccentricity and low inclination in the limiting-mass- and limiting-kinetic-energy-weighted
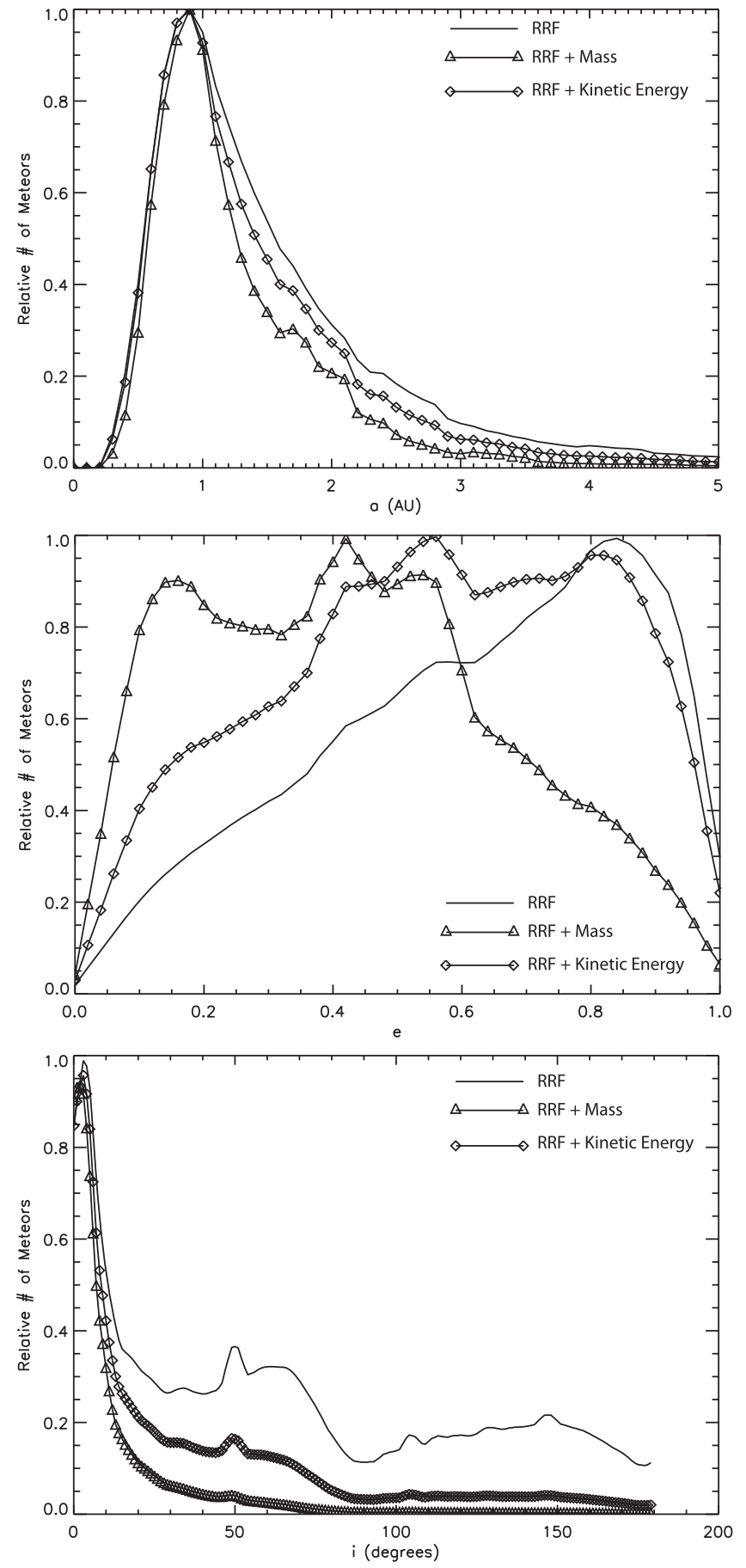

Figure 11. Radar response corrections of heliocentric semimajor axis (top), eccentricity (middle), and inclination (bottom) as determined by SAAMER-OS weighted for limiting trail line density (black), limiting mass (triangle), and limiting kinetic energy (diamond). The bin size is $0.1 \mathrm{AU}, 0.02$, and $1^{\circ}$ for each of the respective quantities.

distributions is consistent with the ZDC model reported by Nesvorný et al. (2010, 2011a) and may also suggest that many of these meteoroids are slow-speed apex meteoroids, which are predicted to provide approximately $20 \%$ of the apex meteoroids (Table 1 in Fentzke \& Janches 2008; Fentzke et al. 2009). Again, these results suggest that meteoroids above a limiting mass are primarily from cometary parent sources and their orbits have significantly decayed from PR drag (Nesvorný et al. 2010, 2011a); however, it is important to note that, 

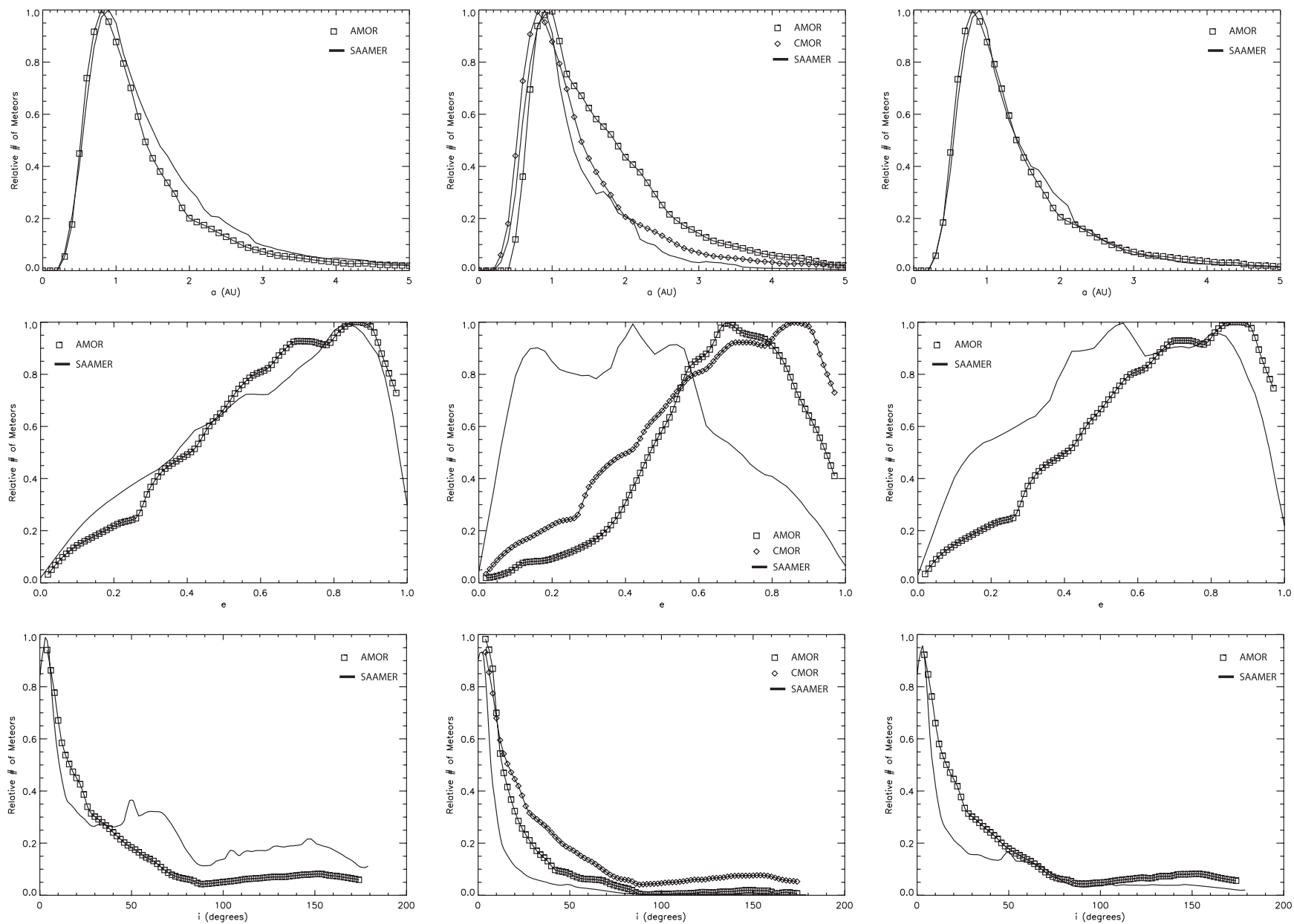

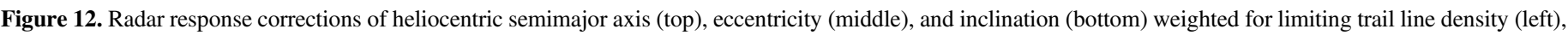

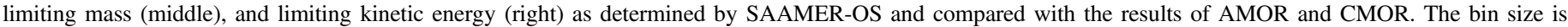
$0.1 \mathrm{AU}, 0.02$, and $1^{\circ}$ for each of the respective quantities.

according to Wiegert et al. (2009), these orbital properties could also suggest asteroidal parent sources. Finally, the strong enhancement seen in the inclination distributions (bottom panel) at $i \approx 55^{\circ}$ agrees with the characteristic inclination of the Southern June Aquilids (SZC) meteor shower (Brown et al. 2008), which has a strong presence in SAAMER-OS data.

Figure 12 shows a comparison of the heliocentric orbital parameter distributions by SAAMER-OS weighted for limiting trail line density, mass, and kinetic energy with those derived with CMOR and AMOR. In the semimajor axis results (top row), the CMOR distribution shows more meteoroids with larger values of $a$ (Campbell-Brown 2008), indicating more meteoroids with larger orbits than the SAAMER-OS results show. This increase is not seen in the AMOR results. Further work may show whether this is related to inter hemispheric asymmetries, since both SAAMER-OS and AMOR sample meteoroids originate from the southern ecliptic sky, as opposed to CMOR, which observed the majority of meteoroids originating from the north. Additionally, owing to the relative transmitting power of these radar systems, SAAMER-OS and AMOR are expected to observe smaller meteoroid masses, which are expected to have orbits more affected by PR drag, resulting in smaller semimajor axis and eccentricity values (Nesvorný et al. 2010, 2011a). It also should be noted that better agreement between the SAAMER-OS and AMOR semimajor axis distributions was found when the SAAMER-
OS trail-line-density- or kinetic-energy-weighted distributions are compared. In particular, for the limiting kinetic energy distribution the agreement is remarkable.

The corrected SAAMER-OS eccentricity distribution weighted for trail line density appears to be consistent with the AMOR results. The only difference between the two distributions is a minor increase in meteoroids with lower eccentricities observed by SAAMER-OS, which is caused by the south toroidal meteoroids, which appear to provide a weaker contribution to AMOR's distributions, although they were not specifically studied by Galligan \& Baggaley (2004, 2005). This difference is more apparent in the kineticenergy-weighted distribution; however, there was still agreement at higher eccentricity values. Comparing the SAAMEROS results weighted for limiting mass with the CMOR distribution, the SAAMER-OS results suggest a dominance of small-semimajor-axis, low-eccentricity meteoroids, whereas the CMOR results indicate a dominance of large-semimajoraxis and high-eccentricity orbits. These outcomes could suggest major differences in the orbital properties of meteoroids in the southern ecliptic sky compared to the northern sky. The results of both systems are consistent with the ZDC model developed by Nesvorný et al. (2010, 2011a), assuming that SAAMER-OS observes 100 times smaller meteoroid masses than CMOR does. This is due to the fact that the minimum detectable mass by a radar is proportional to the square root of 
the transmitted power, and considering that SAAMER transmits about 10 times more power than CMOR (Campbell-Brown 2008). Future plans include simultaneous optical/ radar observations which will allow us to calibrate the radar, and thus obtain mass information for the observed meteors.

In considering the corrected inclination distributions (bottom row), all three systems show a dominance of low-inclination, prograde orbits regardless of the weighting factor. There is reasonable agreement between the SAAMER-OS results weighted for limiting mass and the CMOR distribution (both consistent with the expected properties of helion and antihelion meteoroids). However, the semimajor axis and eccentricity distributions are not in agreement between these systems: the two systems observe different portions of the ZDC. This might show how crucial it is to analyze collectively the orbital parameters in order to draw accurate conclusions about the meteoroid population, and the method to extrapolate their orbit is also important, at least to achieve more precision. Good agreement is also found, between SAAMER-OS and AMOR for the distribution weighted for limiting kinetic energy, with the exception of the increased contribution of toroidal meteoroids observed by SAAMER that caused an increase in the number of high-inclination, prograde orbits. Overall, in most of the distributions considered, the SAAMEROS corrected distributions weighted for limiting kinetic energy resulted in strong agreement with the AMOR results. Unfortunately, AMOR, without weighting for limiting kinetic energy by Galligan \& Baggaley (2004), cannot provide a consistent explanation for the disagreement between its results and the outcomes provided by the other systems weighted for limiting trail line density or limiting mass.

\section{THE SOUTH TOROIDAL SOURCE: A UNIQUE FEATURE OF SAAMER'S OBSERVATIONS}

In this section, the focus is shifted toward the results of meteors originating from the south toroidal source. This is a unique feature of SAAMER-OS's observations given its locations. As demonstrated in the previous sections, the activity from the ST source is relatively strong, representing almost $20 \%$ of the recorded orbits (Table 2). AMOR also could contribute to this, given its optimal location for surveying this source, but no significant results were shown by Galligan \& Baggaley (2004, 2005).

In this section a first look at the ST source is presented, where the events with radiant location with values within $245^{\circ}<\lambda-\lambda_{0}<295^{\circ}$ and $-70^{\circ}<\beta<-50^{\circ}$ are selected. No further selection is performed. Campbell-Brown \& Wiegert (2009) showed that the northern counterpart is composed of at least six different concentrations, not associated with meteor showers, with distinct orbital properties. However, for such a detailed study a better statistical data set should be required, as shown by the authors, and thus it is left for future work. The present study concentrates on a general look at the ST source.

Figure 13 displays the observed geocentric velocity (panel (a)), semimajor axis (panel (b)), eccentricity (panel (c)), and inclination (panel (d)) of those meteors with observed radiants within the ST region defined above. For lack of reported measurements of this source, the SAAMER-OS results are compared with the same quantities from the northern counterpart observed by CMOR and reported by Campbell-Brown (2008). To this purpose, SAAMER's observed distributions corrected by RRF and weighted by a limiting mass in the same manner as CMOR's NT results (Campbell-Brown 2008) are also displayed in the panels. For the case of the geocentric speed, the raw results show a distribution with a peak at $\sim 37$ $\mathrm{km} \mathrm{s}^{-1}$ and a complete absence of particles with velocities greater than $55 \mathrm{~km} \mathrm{~s}^{-1}$. The results from CMOR regarding the NT source are in somewhat agreement with those from SAAMER-OS, although the distribution seems to be shifted toward higher velocities by $\sim 3 \mathrm{~km} \mathrm{~s}^{-1}$. Also, a significant distribution tail of particles with velocities higher than $55 \mathrm{~km} \mathrm{~s}^{-1}$ is recorded. For the case of the corrected distributions, however, some differences emerge. Although the results from both radars show bimodal distribution, the ST's results show a peak at $30 \mathrm{~km} \mathrm{~s}^{-1}$, a dramatic increase of particles with velocities lower than $20 \mathrm{~km} \mathrm{~s}^{-1}$, and a peak at $12 \mathrm{~km} \mathrm{~s}^{-1}$. The NT corrected distribution observed by CMOR peaks at $35 \mathrm{~km} \mathrm{~s}^{-1}$, and while there is an increase in the number of slower particles, the peak is observed at $15 \mathrm{~km} \mathrm{~s}^{-1}$. More importantly, while SAAMER's outputs show a predominance of slower particles that dominate the corrected distribution, CMOR's show the opposite.

In terms of the measured semimajor axis, the raw observations show that both the ST and NT sources peak at $\sim 0.8-0.9$ AU, with the NT sources having a larger portion of particles with $a>2 \mathrm{AU}$ than its southern counterpart. For the corrected distributions, however, a closer agreement between both sources is observed. For the case of the orbital eccentricities, both toroidal sources peak at very small values $(\sim 0.2)$ as compared to the general observed distributions. Both raw and corrected distributions seem to agree between the ST and NT, although the NT seems to have a higher number of meteors with higher eccentricities $(e>0.2)$ as compared to the ST results due to the presence of larger particles in their recorded sample (P. Pokorný 2015, private communication) Finally, some differences between both sources are also seen in the distribution of orbital inclinations, where the ST raw and corrected distribution maxima are $\sim 10^{\circ}$ smaller than their respective distributions of the northern counterpart. Also both NT and ST corrected distributions show an increase of smaller inclination values. However, the ST fraction of these meteors is higher and peaks at significantly lower values $\left(\sim 15^{\circ}\right)$ than the NT does $\left(\sim 35^{\circ}\right)$.

\section{CONCLUSIONS}

In this paper, meteoroid orbital distributions obtained during the three and a half years of operation of the SAAMER-OS were presented. In particular, the distributions of meteoroid speed, radiant, and heliocentric orbital parameters were presented and discussed. The results show that SAAMER-OS is a system capable of providing significant statistical data of meteoroid orbital properties in the largely unexplored southern sky. An effort to fill the observational gap in this region utilizing optical and video observations has taken place in the past few years (Jopek et al. 2010; Bland et al. 2012; Molau \& Kerr 2014; Towner et al. 2015), which focus on larger fireballs and are limited by the weather conditions that optics suffer from. However, SAAMER-OS's survey introduces an important characteristic particular of radar observations: the study of smaller and perhaps more dynamically evolved particles. The observation results from SAAMER-OS were compared with those reported utilizing AMOR (Galligan \& Baggaley 2004, 2005), CMOR (Campbell-Brown 2008), and HRMP (Taylor \& Elford 1998) and were found to be generally in 

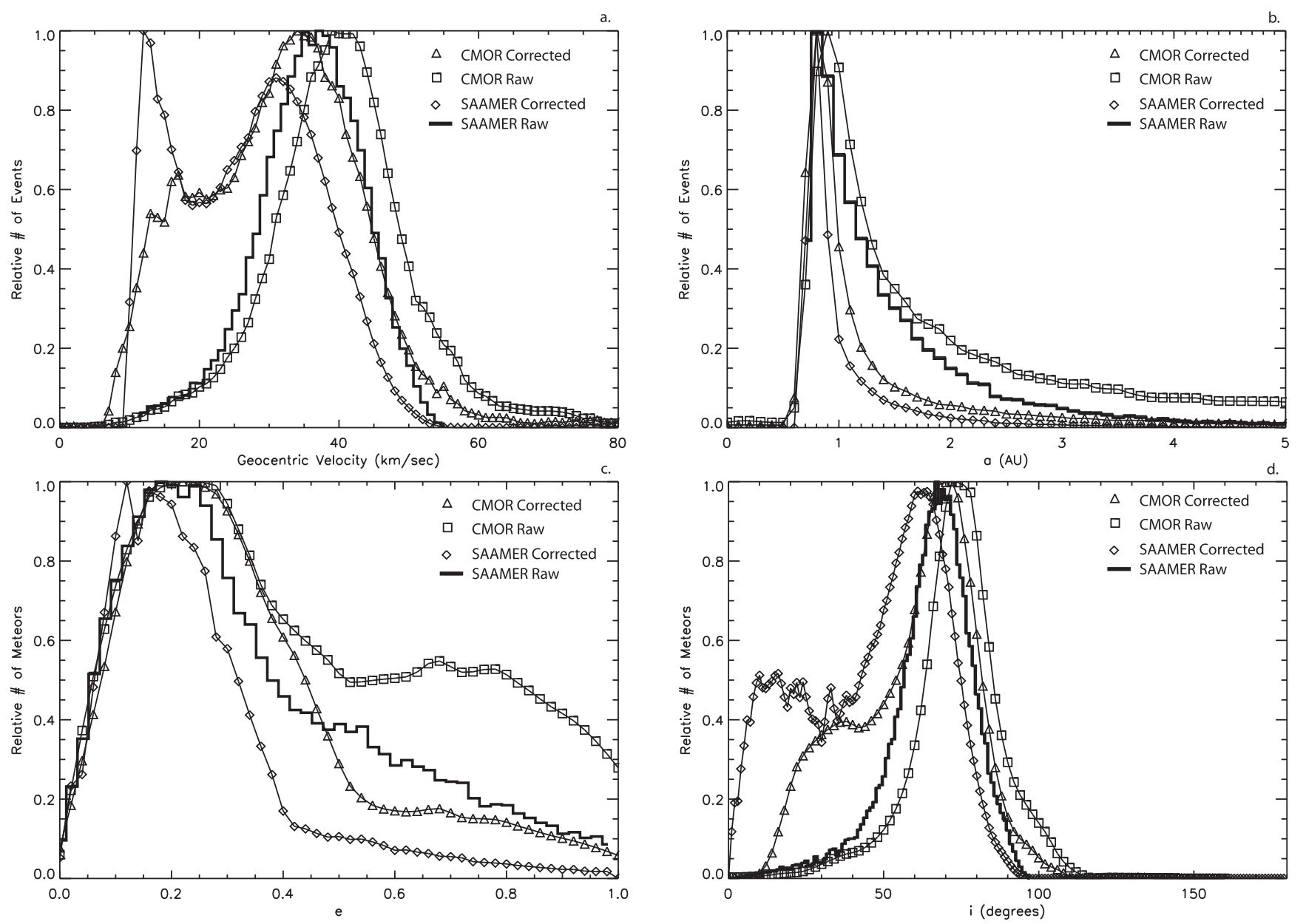

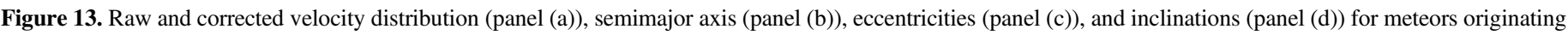

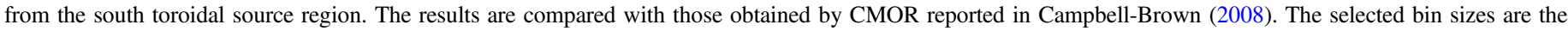
same as in previous plots.

relatively good agreement, providing confidence in this new system and methodology.

The observational biases associated with both the SAAMEROS operating parameters and atmospheric effects have been addressed and characterized using the SAAMER-OS RRF, which provides a weighting factor for each observed meteor based on its probability of detection. The weighting factors were used to correct the observed distributions in order to provide more accurate distributions of meteoroids in the atmosphere within view of SAAMER. These corrected distributions take into account particles that produce meteors with trail electron line density above the SAAMER-OS sensitivity threshold (Ceplecha et al. 1998) independent of whether they were detected by the radar. Additional weighting factors for a constant limiting mass and kinetic energy were also applied, and the bias-corrected distributions derived from the SAAMER-OS observations were presented individually for meteoroids above a minimum detectable trail line density, limiting mass, and limiting kinetic energy.

The SAAMER-OS corrected distributions were again compared to the AMOR, CMOR, and HRMP distributions, resulting in general agreement. It is important to note that the distributions reported for the previous meteor radar surveys had been weighted for a variety of limiting parameters (e.g., minimum line density, limiting mass), demonstrating the need for specifying the limiting parameter for any corrected distributions presented. Comparing, for example, the radar response corrections from SAAMER-OS without additionally weighting for limiting mass with the reported CMOR distributions would lead to erroneous conclusions because they represent different portions of the ZDC. However, when the SAAMER-OS limiting mass distributions were compared with the CMOR and HRMP results, general agreement was found. Concerning AMOR corrections, Galligan \& Baggaley (2004, 2005) did not specify how the results were weighted; however, the SAAMER-OS results weighted for limiting kinetic energy were shown to agree strongly with the reported AMOR results, while those weighted for minimum trail line density (i.e., the radar response correction was applied without any additional weighting) did not agree as well.

These comparisons were made mainly to place the SAAMER-OS in context with previous surveys. Some effects from differences between the systems exist and will be explored in future work. For example, SAAMER-OS uses two remote stations, spaced 13 and $8 \mathrm{~km}$ from the central station. It could then be argued that meteors having very short trail lengths (such as from fragmenting meteoroids) may not be detected at all three stations. This would impose an additional bias where slow, large meteoroids are preferentially detected, skewing the orbital distributions. This could be responsible, in part, for the SAAMER-OS trend displayed in Figure 5. For comparison, the CMOR study reported by Campbell-Brown 
(2008) used two remote stations 6 and $8 \mathrm{~km}$ away, possibly enabling detection of shorter trails. However, the results presented here suggest otherwise, since there is a dominance of high-speed particles, which should be corrected when the radar response correction is applied. The main effect of the remote site distance could be on detection rate: some unobserved high-speed meteoroids.

Regarding the comparisons with the HRMP results, since this system had no interferometer capabilities, velocities were derived from the Fresnel diffraction pattern (Ceplecha et al. 1998). This method biases the observed distributions against fragmenting meteoroids, which may be significant for higher-speed meteors.

There is also an issue for comparisons with the AMOR system. While AMOR had less transmitting power than SAAMER, the receiver array used a pencil beam (not allsky), leading to a much lower mass limit. The work presented here assumes a power-law distribution that is equal for all the sporadic sources, but if this is not true, then the AMOR results may not be directly comparable. The pencil-beam radiation pattern may have also been responsible, at least in part, for the inability of AMOR to observe the ST sources as it limits the radiants that are observable by the system.

Some conclusions regarding the choice of limiting parameter used to weight the corrected meteoroid distributions must be made. Meteoroid distributions are often reported for particular mass thresholds, as is the case for CMOR and HRMP, as well as for some high-power, large-aperture radar systems (Janches et al. 2014b). Additionally, the characteristics of many meteoroid models (e.g., the ZDC model; Nesvorný et al. 2010, 2011a) are reported using limiting mass thresholds. While the choice of limiting mass is an understandable one based on its physical significance, it is important to note that these distributions tend to misrepresent the meteoroid population observed by ground-based systems. Because the weighting for the very few slow-speed meteoroids is two orders of magnitude greater than the more commonly observed highspeed meteoroids, the limiting mass distributions are dominated by the modeled speed profile used in the limiting mass weighting factor. Furthermore, the meteoroid speed is highly correlated with its radiant and heliocentric orbital properties, and so weighting for limiting mass has large effects on these distributions as well. Moreover, the results of weighting for limiting mass are really only representative of meteoroids with masses greater than the minimum detectable mass at the slow speeds. Otherwise, an assumption is made that meteoroid properties have constant distributions at all masses, which is unlikely as the smaller meteoroid masses probably come from entirely different parent sources and parts of the solar system compared to the larger meteoroid masses (Nesvorný et al. 2010, 2011a, 2011b; Pokorný et al. 2014). Thus, the effect of using the limiting mass weighting essentially removes a significant percentage of the observed data from the results, reducing the overall significance of ground-based observations.

The limiting mass distribution is also not the most useful for many applications of meteoroid research. For meteoric mass depositions in the atmosphere, estimating the amount of ablated material is highly dependent on particle speed (Janches et al. 2014b) and is perhaps better represented by the meteoroid's ionization efficiency or kinetic energy. For these applications, weighting for minimum trail line density or limiting kinetic energy is likely more directly applicable. For space applications in which the major concern is meteoroid collisions with spacecraft, the limiting mass distribution is also not the best choice. The energy of the impactor and the charge production of resulting collisions are the major drivers for failure risk (Close et al. 2010). Thus, for spacecraft safety concerns, the limiting kinetic energy and the minimum line density distributions are more relevant as well. Moreover, the distributions weighted for these factors are much more representative of the radar observations themselves, and even if the limiting mass distributions are true, they are not good representations of the properties of meteoroids that interact with the atmosphere or present impact threats to spacecraft and/or artificial satellites.

In addition, SAAMER-OS is very effective at detecting meteors originating from the south toroidal source, of which limited observational data are available to date. Thus, the far mid-southern latitude of SAAMER-OS makes it a system capable of providing unique observations of a specific portion of the meteoroid population in critical need of further exploration. An initial study of the orbital characteristics of this source was reported in this work. It is important to note that in order to obtain the orbital information, we have applied a patched conic methodology, which, although acceptable, is not as accurate as orbital numerical integration. For lack of data from previous surveys, a comparison with results from its northern counterpart was also presented. Although general agreement was found, some differences between both were identified. For example, our results show some disagreement with Pokorný et al. (2014). They found that the long-term stable part of the north toroidal source is mainly fed by dust released by Halley-type (long-period) comets. According to their results, the southern counterpart should be symmetric. Furthermore, Campbell-Brown \& Wiegert (2009) showed that the NT source is composed of 12 broad radiant concentrations, separated by either time or radiant location. Six of these enhancements are broad distributions associated with more focused shower activity, while the other six are not associated with major showers. Four of these sporadic concentrations have constant location at the north toroidal center, while the other two appear to drift toward the helion and antihelion sources. The disagreement between the north and south toroidal sources found in our work can be due to differences in the basic source structure. The results reported by Campbell-Brown \& Wiegert (2009) were possible thanks to the high number of orbits obtained by a decade of observations performed by CMOR, which enable high-resolution studies. This emphasizes the importance of continuing the operation of SAAMER-OS; more detailed studies will be reported in the future when a sufficient number of orbits is obtained.

Finally, we want to underline further that SAAMER-OS offers similarly unique capabilities for future studies of meteor showers and streams given the range of ecliptic latitudes that the system is able to survey. Radiant ecliptic latitudes can be observed from the ecliptic south pole to approximately $30^{\circ} \mathrm{N}$. Therefore, future surveys will be able to study in detail showers at high southern latitudes (e.g., July Phoenicids or Puppids complex) that, for example, are unobservable from northern radars such as CMOR.

The deployment of SAAMER and its remote receiving stations, as well as the work presented in this paper, were 
supported by NSF awards AGS-0634650, AGS-0944104, and AST-0908118. D.J.'s participation is currently supported through NASA awards 12-PAST12-0007 and 12-PATM120006. The authors wish to give special thanks to the EARG personnel for their invaluable assistance with the operation and day-to-day oversight of SAAMER. Without their help, operating a system on the other side of the planet would be impossible! The authors wish to thank Prof. Peter Brown for invaluable guidance for the final SAAMER-OS design and deployment. Finally, the authors wish to acknowledge S. Pifko for his work in determining SAAMER-OS orbits. Unfortunately, following editorial rules, we could not include him as an author because, despite numerous efforts to communicate with him, he has stopped answering us and did not confirm his approval to be included as an author.

\section{REFERENCES}

Baggaley, W. 2002, in Meteors in the Earth's Atmosphere, ed. E. Murad \& I. Williams (Cambridge: Cambridge Univ. Press)

Baggaley, W., Bennett, R., Steel, D., \& Taylor, A. 1994, QJRAS, 35, 293

Baggaley, W. J. 1980, BAICz, 31, 308

Bate, R. R., Mueller, D. D., \& White, J. E. 1971, Fundamentals of Astrodynamics (New York: Dover)

Backman, D. 1997, Exozody Workshop, Extrasolar Zodiacol Emission: NASA Study Panel Report (Mountain View, CA: NASA-Ames)

Beichman, C. A. 1987, ARA\&A, 25, 521

Bland, P. A., Spurný, P., Bevan, A. W. R., et al. 2012, AuJES, 59, 177

Bronshten, V. 1983, Physics of Meteoric Phenomena (Dordrecht: Reidel)

Brown, P., Jones, J., Weryk, R. J., \& Campbell-Brown, M. D. 2004, EM\&P, 95, 617

Brown, P., Weryk, R. J., Wong, D. K., \& Jones, J. 2008, EM\&P, 102, 209

Brown, P., Wong, D. K., Weryk, R. J., \& Wiegert, P. 2010, Icar, 207, 66

Campbell-Brown, M., \& Wiegert, P. 2009, M\&PS, 44, 1837

Campbell-Brown, M. D. 2008, Icar, 196, 144

Ceplecha, Z., Borovička, J., Elford, W., et al. 1998, SSRv, 84, 327

Close, S., Brown, P., Campbell-Brown, M., Oppenheim, M., \& Colestock, P. 2007, Icar, 186, 547

Close, S., Colestock, P., Cox, L., Kelley, M., \& Lee, N. 2010, JGRA, 115, A12328

Close, S., Hunt, S., Minardi, M., \& McKeen, F. 2000, RaSc, 35, 1233

Cziczo, D., Thomson, D., \& Murphy, D. 2001, Sci, 291, 1772

Drolshagen, G., Dikarev, V., Landgraf, M., Krag, H., \& Kuiper, W. 2008 EM\&P, 102, 191

Feng, W., Marsh, D. R., Chipperfield, M. P., et al. 2013, JGRD, 118, 9456

Fentzke, J. T., \& Janches, D. 2008, JGRA, 113, A03304

Fentzke, J. T., Janches, D., \& Sparks, J. J. 2009, JASTP, 71, 653

Fritts, D. C., Janches, D., \& Hocking, W. K. 2010a, JGRD, 115, 19123

Fritts, D. C., Janches, D., Hocking, W. K., Mitchell, N. J., \& Taylor, M. J. 2012, JGRD, 117, 10108

Fritts, D. C., Janches, D., Iimura, H., et al. 2010b, JGRD, 115, 18112

Gabrielli, P., Barbante, C., ad Plane, J., Varga, A., et al. 2004, Natur, 432, 1011

Galligan, D. P., \& Baggaley, W. J. 2004, MNRAS, 353, 422
Galligan, D. P., \& Baggaley, W. J. 2005, MNRAS, 359, 551

Gardner, C. S., Chu, X., Espy, P., et al. 2011, JGRD, 116, D02304

Hawkins, G. S. 1963, SCoA, 7, 53

Hocking, W. K., Fuller, B., \& Vandepeer, B. 2001, JATP, 63, 155

Hocking, W. K., Thayaparan, T., \& Jones, J. 1997, GeoRL, 24, 2977

Hughes, D. W. 1978, in Cosmic Dust, ed. J. A. M. McDonnell (Chichester: John Willey)

Hunt, S., Oppenheim, M., Close, S., et al. 2004, Icar, 168, 34

Janches, D., Hocking, W., Pifko, S., et al. 2014a, JGRA, 119, 2269

Janches, D., Nolan, M., Meisel, D., et al. 2003, JGR, 108, 1222

Janches, D., Plane, J. M. C., Nesvorný, D., et al. 2014b, ApJ, 796, 41

Jenniskens, P. 2006, Meteor Showers and Their Parent Comets (Cambridge: Cambridge Univ. Press)

Jenniskens, P. 2008, EM\&P, 102, 505

Jenniskens, P., Gural, P. S., Dynneson, L., et al. 2011, Icar, 216, 40

Jones, J., \& Brown, P. 1993, MNRAS, 265, 524

Jones, J., Brown, P., Ellis, K. J., et al. 2005, P\&SS, 53, 413

Jopek, T. J., Koten, P., \& Pecina, P. 2010, MNRAS, 404, 867

Lanci, L., \& Kent, D. V. 2006, GeoRL, 33, 13308

Lanci, L., Kent, D. V., \& Biscaye, P. E. 2007, GeoRL, 34, 10803

Lau, E. M., Avery, S. K., Avery, J. P., et al. 2006, RaSc, 41, 4007

Love, S., \& Brownlee, D. E. 1993, Sci, 262, 550

Marsh, D., Janches, D., Feng, W., \& Plane, J. 2013, JGRD, 118, 11442

Mathews, J. D., Janches, D., Meisel, D., \& Zhou, Q. 2001, GeoRL, 28, 1929

McKinley, D. W. R. 1961, Meteor Science and Engineering (New York: McGraw-Hill)

Molau, S., \& Kerr, S. 2014, WGN JIMO, 42, 68

Nesvorný, D., Janches, D., Vokrouhlický, D., et al. 2011a, ApJ, 743, 129

Nesvorný, D., Jenniskens, P., Levison, H. F., et al. 2010, ApJ, 713, 816

Nesvorný, D., Vokrouhlický, D., Pokorný, P., \& Janches, D. 2011b, ApJ, 743, 37

Peregudov, F. I. 1958, SvA, 2, 833

Picone, J. M., Hedin, A. E., Drob, D. P., \& Aikin, A. C. 2002, JGRA, 107,1468

Plane, J. 2003, ChRv, 103, 4963

Plane, J. 2004, ACP, 4, 39

Plane, J., Feng, W., \& Dawkins, E. 2015, ChRv, 115, 4497

Plane, J. M. C. 2012, Chemical Society Reviews, 41, 6507

Pokorný, P., Vokrouhlický, D., Nesvorný, D., Campbell-Brown, M., \& Brown, P. 2014, ApJ, 789, 25

Sekanina, Z. 1973, Icar, 18, 253

Sekanina, Z. 1976, Icar, 27, 265

Steel, D. I., \& Elford, W. G. 1991, JATP, 53, 409

Taylor, A., \& Elford, W. 1998, EP\&S, 50, 569

Towner, M. C., Bland, P. A., Cupak, M. C., et al. 2015, in Lunar and Planetary Inst. Tech. Rep. 46, Lunar and Planetary Science Conf. (Woodland, TX: LPI), 1693

Verniani, F. 1973, JGR, 78, 8429

Vondrak, T., Plane, J. M. C., Broadley, S., \& Janches, D. 2008, ACP, 8, 7015

Wasson, J., \& Kyte, F. 1987, GeoRL, 14, 779

Webster, A. R., Brown, P. G., Jones, J., Ellis, K. J., \& Campbell-Brown, M. 2004, ACPD, 4, 1181

Weryk, R. J., \& Brown, P. G. 2013, P\&SS, 81, 32

Wiegert, P., Vaubaillon, J., \& Campbell-Brown, M. 2009, Icar, 201, 295

Wiegert, P. A., Brown, P. G., Weryk, R. J., \& Wong, D. K. 2011, MNRAS, 414, 668

Wiesel, W. 1997, Spaceflight Dynamics (New York: Mcgraw-Hill) 